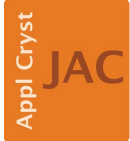

JOURNAL OF

APPLIED

CRYSTALLOGRAPHY

ISSN 1600-5767

Received 1 September 2019

Accepted 19 April 2020

Edited by V. T. Forsyth, Institut Laue-Langevin, France, and Keele University, UK

Keywords: grazing-incidence wide-angle X-ray scattering; GIWAXS; organic materials.

Supporting information: this article has supporting information at journals.iucr.org/j

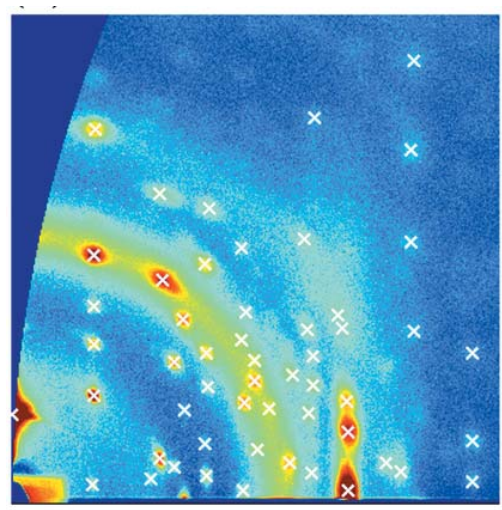

(C) 2020 International Union of Crystallography

\section{GIWAXS-SIIRkit: scattering intensity, indexing and refraction calculation toolkit for grazing-incidence wide-angle $X$-ray scattering of organic materials}

\author{
Victoria Savikhin, ${ }^{\mathrm{a}, \mathrm{b}}$ Hans-Georg Steinrück, ${ }^{\mathrm{a}, \mathrm{c}}$ Ru-Ze Liang, ${ }^{\mathrm{d}}$ Brian A. Collins, \\ Stefan D. Oosterhout, ${ }^{a}$ Pierre M. Beaujuge ${ }^{d}$ and Michael F. Toney ${ }^{a *}$
}

\begin{abstract}
a Stanford Synchrotron Radiation Lightsource, SLAC National Accelerator Laboratory, 2575 Sand Hill Road, Menlo Park, CA 94025, USA, 'belectrical Engineering Department, Stanford University, 350 Serra Mall, Stanford, CA 94305, USA,

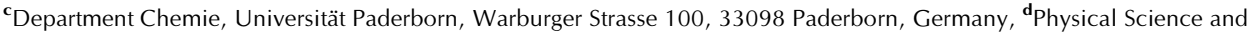
Engineering Division, KAUST Solar Center, King Abdullah University of Science and Technology (KAUST), Thuwal 23955-6900, Saudi Arabia, and ' Department of Physics and Astronomy, Washington State University, Pullman, WA 99164, USA. *Correspondence e-mail: mftoney@slac.stanford.edu
\end{abstract}

Grazing-incidence wide-angle X-ray scattering (GIWAXS) has become an increasingly popular technique for quantitative structural characterization and comparison of thin films. For this purpose, accurate intensity normalization and peak position determination are crucial. At present, few tools exist to estimate the uncertainties of these measurements. Here, a simulation package is introduced called GIWAXS-SIIRkit, where SIIR stands for scattering intensity, indexing and refraction. The package contains several tools that are freely available for download and can be executed in MATLAB. The package includes three functionalities: estimation of the relative scattering intensity and the corresponding uncertainty based on experimental setup and sample dimensions; extraction and indexing of peak positions to approximate the crystal structure of organic materials starting from calibrated GIWAXS patterns; and analysis of the effects of refraction on peak positions. Each tool is based on a graphical user interface and designed to have a short learning curve. A user guide is provided with detailed usage instruction, tips for adding functionality and customization, and exemplary files.

\section{Introduction}

Interest in the science behind semiconducting organic materials has grown considerably as organic-based electronics enter mainstream markets (Chen et al., 2019). Advanced characterization of material packing structure and disorder is necessary to better understand structure-function relationships. Traditional characterization approaches that are widely used for inorganic materials are not as well suited for organic materials due to their faster degradation and inherently large disorder. For this reason, X-ray techniques have been used extensively. On the length scale of about 1-30 A, wide-angle $\mathrm{X}$-ray scattering (WAXS) is a popular approach, with grazingincidence WAXS (GIWAXS) maximizing the signal strength (Liu et al., 2013). For similar reasons, GIWAXS has also become popular in understanding perovskite thin films (Lilliu et al., 2016; Chen et al., 2018; Zhong et al., 2018). Other nanoscale thin-film materials benefit from GIWAXS as well: a few examples include polyethylene (Sasaki et al., 2007), porphyrins (Liman et al., 2013; Kim, Lee et al., 2012), block copolymers (Liu et al., 2012; Lin et al., 2014) and nanoparticlebased superlattices (Corricelli et al., 2014).

Peak positions in a GIWAXS pattern provide information about reciprocal-lattice dimensions and symmetry, while the 
intensity of peaks can be used as a measure of the relative degree of crystallinity (Hernandez et al., 2017) or the amount of mixed phase in a blend (Oosterhout et al., 2017; Ran et al., 2017). In well ordered materials with many observable scattering peaks, a crystal structure can be obtained from a single GIWAXS pattern (Servet et al., 1993; Akimichi et al., 1993; Sherman et al., 2015; Nabok et al., 2007). The relative intensities of peaks versus incident angle can give clues to the vertical stratification within a film (Gann et al., 2018). In addition, the width and/or shape of peaks can be used to find structural coherence lengths (Noriega et al., 2013; Rivnay et al., 2011).

Accurate measurements and meaningful comparisons of material characteristics require an understanding of inherent uncertainties in a typical GIWAXS setup. In many cases, changing the instrumental setup can affect a scattering pattern in a complex nonlinear fashion (Toney \& Brennan, 1989; Klockenkàmper et al., 1992; Müller-Buschbaum, 2014). A major factor contributing to this is the incident beam's penetration depth and waveguiding within a thin film, which is subject to interference effects. Another consideration is the conversion of a detector image to a usable reciprocal-space pattern (with axes of $Q_{x y}$ and $Q_{z}$, which are the components of the scattering vector along the substrate and normal to the substrate, respectively), for which a simple analytical relationship does not exist (Stribeck \& Nöchel, 2009). Towards this end, we have developed a suite of simulation tools that will help GIWAXS users to estimate uncertainties and necessary corrections involved in a given measurement, described in Sections 3, 4, 5 and 6. We have also developed a toolset for estimating the molecular packing, described in Sections 7 and 8. Each standalone tool runs in MATLAB and consists of a graphical user interface (GUI) with straightforward user inputs. The outputs are designed to answer user questions intuitively with a short learning curve. The software package is currently designed to import images that have been converted to $Q_{x y}-Q_{z}$ space with all relevant corrections applied, as explained below.

Our first tool, described in Section 3, is a standalone complex refractive index calculator. The refractive index of a material is an important parameter used in other calculations.

Section 4 describes a tool for calculating relative changes to scattering intensity arising from differences in sample geometry or setup geometry. This tool considers two factors: the lateral beam footprint (or beam projection, taking into account spillover) on the sample and the penetration of an $\mathrm{X}$-ray beam into a thin film. The X-ray penetration is estimated using the Parratt formalism (Parratt, 1954; Dev et al., 2000). Together, these effects give rise to an effective scattering volume which can be used to normalize data from samples with varying incident angles, sample dimensions and/ or composition. Due to the non-constant spatial intensity distribution in the incident beam, we calculate the fraction of the beam intensity that is incident on the sample rather than the irradiated area. To avoid confusion, we refer to the effective scattering volume as a 'normalization factor'.

The tool in Section 4 can also calculate uncertainties/ confidence intervals in the normalization factors given uncertainties in setup geometry. Publications often report GIWAXS results with approximate normalization (e.g. dividing by film thickness) and without uncertainties due to the complexity of this calculation. Our software will help users understand and confidently interpret their GIWAXS results, making this method more attractive. Section 5 provides an example calculation of the normalization factor and uncertainties for a hypothetical sample set using the tool introduced in Section 4.

The next tool, described in Section 6, calculates the effect of refraction on the observed location of scattering peaks for thin films. The corresponding peak shift has been previously calculated for the out-of-plane scattering direction (Toney \& Brennan, 1989) and for peaks in the small-angle regime (Busch et al., 2011). Here, we extend and apply these calculations to off-axis peaks in the wide-angle regime. We also evaluate the position of an additional peak which arises due to reflection off the substrate. This effect was previously examined for a thin film of $2,2^{\prime}: 6^{\prime}, 2^{\prime \prime}$-ternaphthalene by Resel et al. (2016); we compare our results with those of Resel et al. (2016) in Appendix $B$. In cases where two or more similarly positioned peaks are visible, our tool can help distinguish whether these arise from reflection effects or from scattering of multiple polymorphs.

Section 7 presents a set of tools which allows for peak position extraction and unit-cell calculation in a user-friendly way. Other software packages exist to index GIWAXS patterns, but these can be non-intuitive and difficult to apply to less well ordered materials such as organics. For example, GSAS (Toby \& Dreele, 2013; Giri et al., 2011) fits diffraction peaks to an arbitrary crystal structure symmetry, but the indexing is limited to powder diffraction and thus does not provide any information about orientation. Furthermore, due to the often textured nature of thin films, an integrated $I(Q)$ distribution from a GIWAXS pattern may have different peak intensities compared with a true powder pattern. Another software package is GIXSGUI (Jiang, 2015), which allows visualization of the diffraction pattern from a crystal structure overlaid with the data, but requires the user to input unit-cell parameters. This may be possible for structures close to cubic or when the structure can be accurately guessed from simulation, but this is not the case for many organic materials. Similarly, SimDiffraction (Breiby et al., 2008) simulates a grazing-incidence diffraction pattern from a molecule and crystal structure with relative peak intensities, but the user is required to have prior knowledge of the molecular packing. On the other hand, the Diffraction Pattern Calculator (DPC) toolkit (Hailey et al., 2014) performs an elegant calculation of crystal structure given a GIWAXS pattern without userspecified parameters. The major drawback of the DPC toolkit is that it requires well defined scattering peaks from a singlecrystal structure without large background signal. In contrast, some materials such as organic photovoltaic (OPV) films may exhibit a large amorphous halo, contain multiple polymorphs or contain a blend of multiple materials with overlapping scattering. For example, the small molecules analyzed in Section 8 crystallize poorly unless blended with [6,6]-phenyl-C71-butyric 
acid methyl ester $\left(\mathrm{PC}_{71} \mathrm{BM}\right)$, and one of the small molecules is demonstrated to form at least two polymorphs.

Our indexing toolset does not assume prior knowledge of material packing. As described in Section 7, the tool can import patterns generated by wxdiff (.tif format) (Mannsfeld et al., 2011) or WAXStools (.bin format) (Oosterhout et al., 2017), and the peak fitting routine works with the user to isolate peaks from the background. Suggestions for adapting the tool to other image formats are given in the user guide; however, it is assumed that all necessary corrections from the raw detector image to $Q_{x y}-Q_{z}$ space have already been applied, as described below. The user can also choose to import a list of peak positions rather than a GIWAXS pattern, which makes it possible to separate peaks from different polymorphs. In Section 8, we walk through the usage of the toolset described in Section 7 for a set of two small molecules previously studied for OPVs.

It must be noted that several corrections to the recorded pixel intensities must be applied when 2D detectors are utilized. These include inter alia polarization correction and solid-angle correction. For a detailed discussion we refer the reader to the work of Skinner et al. (2012). In addition, and specific for GIWAXS, each pixel is typically re-meshed onto an equal-spacing $2 \mathrm{D}$ grid where each pixel has an in-plane scattering vector component $Q_{x y}$ and an out-of-plane scattering vector component $Q_{z}$. This is in contrast to traditional transmission powder diffraction where only the magnitude of the scattering vector $\left[Q=\left(Q_{x y}^{2}+Q_{z}^{2}\right)^{1 / 2}\right]$ is of interest. The $Q_{x y}-Q_{z}$ map allows for in-depth analysis of film properties referenced to the substrate orientation (e.g. edge-on versus face-on orientation of polymers). Several software packages (Jiang, 2015; Dane et al., 2020; Mannsfeld et al., 2011; Oosterhout et al., 2017) already exist for this purpose, which is why our software uses the $Q_{x y}-Q_{z}$ arrays as the starting point for subsequent analysis. We point out that the relevance for the importance of these corrections depends on the details of each experiment and experimental setup. A general rule of thumb is that most corrections become more significant with increased scattering angles (Skinner et al., 2012).

The software discussed in this article is freely available for download from https://www-ssrl.slac.stanford.edu/toneygroup. The software is open source and contains extensive commenting. We include a user guide with detailed instructions for usage and tips for customizing the calculations.

\section{Suggested workflow}

The GIWAXS-SIIRkit is designed as a collection of tools that can be used together or separately, depending on the scientific question of a particular experiment. We show a proposed workflow for how the tools can be used in conjunction in Fig. 1.
First, the complex refractive index is found from the chemical structure and density; this is used in several other tools. Next, the peak intensities and positions are extracted from the $Q_{x y}-$ $Q_{z}$ scattering pattern. The peak intensities can be used as estimates of the degree of crystallinity, but first the scattering intensity tool is used to calculate the appropriate normalization factor and uncertainties. The peak positions are corrected for refraction, and then they can be used to either estimate $d$ spacings directly or to calculate a crystal structure.

\section{Calculation of the complex refractive index}

Several of the scattering effects discussed throughout this article depend on the complex refractive index of the film and the substrate, $n=1-\delta+i \beta$. Here, we use the convention of the $z$ coordinate becoming more negative deeper into the substrate. The complex refractive index (Watts, 2014; Attwood, 1999) far from resonance energies can be calculated using

$$
\begin{aligned}
& \delta=\frac{N_{\mathrm{A}} r_{\mathrm{e}} \lambda^{2}}{2 \pi} \frac{\rho}{M_{\mathrm{W}}} f_{1}^{\text {molecule }} \simeq \frac{N_{\mathrm{A}} r_{\mathrm{e}} \lambda^{2}}{2 \pi} \frac{\rho Z}{A}, \\
& \beta=\frac{N_{\mathrm{A}} r_{\mathrm{e}} \lambda^{2}}{2 \pi} \frac{\rho}{M_{\mathrm{W}}} f_{2}^{\text {molecule }}=\frac{\lambda \mu}{4 \pi},
\end{aligned}
$$

where $N_{\mathrm{A}}$ is Avogadro's number, $M_{\mathrm{W}}$ is the molecular weight, $\mu$ is the attenuation coefficient, $r_{\mathrm{e}}$ is the classical electron radius, $f_{1,2}$ are components of the complex scattering factor for the forward direction, given as $f_{1}+i f_{2}, Z$ is the average atomic number, $\lambda$ is the $\mathrm{X}$-ray wavelength, $A$ is the average atomic weight and $\rho$ is the mass density.

Since the complex refractive index is useful for a variety of calculations, we present a simple tool to calculate refractive indices for convenience. Our calculator is similar to the one available online from the Center for X-Ray Optics (CXRO): we sum atomic scattering factors retrieved from their web site (Henke et al., 1993) to approximate molecular scattering factors. Both $\beta$ and $\delta$ are proportional to $\rho$, so an accurate measurement of $\rho$ is necessary for optimal accuracy. In organic materials, $\rho$ can depend on processing conditions, so a 
(a)
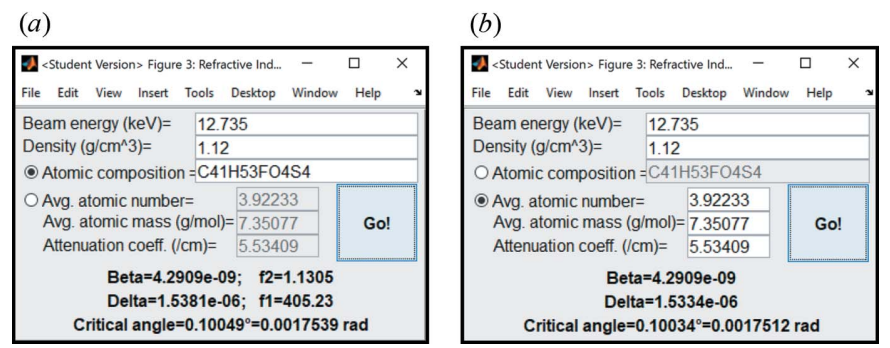

Figure 2

Complex refractive index calculator MATLAB interface, using the polymer PTB7 as an example. (a) Result of calculation using the exact chemical formula; $(b)$ exact $\beta$ and approximate $\delta$ result given the average atomic number, mass and attenuation coefficient of PTB7.

measurement of the sample used in experiments rather than a table lookup is preferable. Our tool can also use $\mu, Z$ and $A$ to calculate $\beta$ and approximate $\delta$ if desired. In addition, our version supports parenthesis grouping (more on this below), which can make it easier to calculate the refractive index for a blend of materials.

The interface for this tool is shown in Fig. 2 for the widely used polymer PTB7 $\left(\mathrm{C}_{41} \mathrm{H}_{53} \mathrm{FO}_{4} \mathrm{~S}_{4}\right)$. The atomic composition should be written in the form ' $X x 1 Y y 2 Z 3 \ldots$ ', where $X x, Y y$, $Z$ are atomic identifiers consistent with the periodic table and $1,2,3$ are the quantities of respective atoms (these can be decimal numbers, e.g. 1.23). Use of parenthesis grouping is also supported, including nested parentheses. For example, $(\mathrm{CH} 2) 3$ is the same as $\mathrm{C} 3 \mathrm{H} 6$ and $((\mathrm{CH} 2) 3 \mathrm{CH} 3) 2$ is the same as $\mathrm{C} 8 \mathrm{H} 18$. Spaces, commas and other symbols can be added to improve legibility; these are ignored in the calculation. In Fig. 2(a), we show the exact result using atomic composition, while Fig. 2(b) shows the $\delta$ value estimated from $Z$ and $A$. Table S1 (in the supporting information) gives the results for some materials typically used in OPVs for $12.7 \mathrm{keV}$ X-rays. For typical materials without higher atomic number elements (e.g. zinc in $\mathrm{ZnPC}$ ), the attenuation coefficient $\mu$ is on the order of $1-10 \mathrm{~cm}^{-1}, \beta=(0.5-7) \times 10^{-9}, \delta=(1.4-2.2) \times 10^{-6}$ and the $\delta$ approximation is accurate to within $0.4 \%$. Our calculator also displays the critical angle which is $(2 \delta)^{1 / 2} \mathrm{rad}$.

Calculating the refractive index close to resonance requires a more robust approach involving a measurement of the absorption spectrum. This process is described by Savikhin \& Toney (2019).

\section{Calculation of normalization factors and uncertainties in scattering intensity}

GIWAXS is an essential measurement for comparing the degree of crystallinity between samples, which is especially important for calculating the amount of mixed phase in organic blends (Oosterhout et al., 2017; Savikhin, Babics et al., 2018). Direct comparison of the scattering intensity from several samples requires accurate normalization and uncertainty analysis. In this section, we present a tool for calculating the normalization factors for a sample set along with uncertainties. By using our software prior to a measurement, users can determine the setup that will yield the most accurate results and the highest scattering intensity (via optimal choice of sample dimensions, incident angle etc.). After data collection, our tool can help determine normalization factors and uncertainties.

In addition to the degree of crystallinity, the scattering intensity arising from a given sample depends on the incoming beam intensity, the fraction of the beam that lands on the sample (versus spilling over the edges), the effective scattering volume and the exposure time. Therefore, obtaining a number representing the degree of crystallinity requires normalizing the measured data by these factors. Geometric considerations will determine the beam footprint $F_{\text {footprint }}$. Optical properties of the materials will determine the propagation of the incident beam into the film: specifically, we look at the integrated electric field across the thickness of the film, $\int_{0}^{T}|E(z)|^{2} \mathrm{~d} z$ (where $T$ is thickness), to estimate scattering volume. Altogether, in order to accurately compare GIWAXS results from different samples, measured intensities need to be normalized (i.e. divided) by a normalization factor $N$, which is given by

$$
N=I_{0} F_{\text {footprint }}\left[\int_{0}^{T}|E(z)|^{2} \mathrm{~d} z\right] t .
$$

Here $I_{0}$ is a measurement representing the incoming beam intensity, which is usually given in arbitrary units such as detector counts, and $t$ is exposure time. $F_{\text {footprint }}$ depends on the incident angle, sample and beam dimensions, and sample position. The electric field in the film depends on incident angle, beam energy, beam divergence, sample density, sample and substrate refractive indices, and sample thickness. More details on the calculation of $F_{\text {footprint }}$ and $\int_{0}^{T}|E(z)|^{2} \mathrm{~d} z$ are given in Sections 4.1 and 4.2, respectively. While normalizing by beam intensity and time is straightforward, the footprint and electric field correction can affect the normalization in a nonlinear and non-intuitive fashion. Thus, the first purpose of our scattering intensity tool is to provide an analysis of how these two quantities change with the measurement setup and, in particular, what their contribution to the normalization will be for a set of samples. The sample dimensions, thicknesses and compositions are especially likely to vary within a set of samples. For this purpose, we define a normalization factor $N_{\text {factor }}=F_{\text {footprint }} \int_{0}^{T}|E(z)|^{2} \mathrm{~d} z$ (here, $\left.N=N_{\text {factor }} I_{0} t\right)$.

A screenshot of the scattering intensity analysis tool interface is shown in Fig. 3(a) and some of the variable definitions are shown in Fig. $3(b)$. The $X$-axis and $Y$-axis dropdown menus specify the output mode:

(i) Both axes assigned to variables: $N_{\text {factor }}$ will be shown as a 2D image, where the image color scale corresponds to the magnitude of $N_{\text {factor }}$ and the axes are as specified.

(ii) Only $X$ axis assigned to a variable ( $Y$ axis set to 'none'): $N_{\text {factor }}$ will be shown as a $1 \mathrm{D}$ plot, where the $Y$ axis corresponds to the magnitude of $N_{\text {factor }}$.

(iii) Both axes set to 'none': initializes the uncertainty calculation mode.

Each variable has an input box for the nominal value. The variable(s) that are chosen as an $X$ or $Y$ axis also have a \pm box enabled, which specifies the range of interest. For example, an 
incident angle $\theta_{0}$ of $0.12 \pm 0.005^{\circ}$ [as shown in Fig. 3(a)] will display $N_{\text {factor }}$ calculated for $\theta_{0}$ ranging from 0.115 to $0.125^{\circ}$. The spatial beam shape is selected from a dropdown menu (for details refer to Section 4.1). The refractive index of the substrate can be entered manually, or the 'Select substrate' dropdown menu automatically fills in the substrate $\beta$ and $\delta$ calculated for a selection of common substrates at an incident energy of $12.7 \mathrm{keV}$ (as shown in Table S1). The purpose of the 'Enter sample list...' button will be discussed in Section 5.

If sample density is one of the variables, it is assumed that the nominal sample $\beta$ and $\delta$ correspond to the nominal density value given, and they are adjusted to follow changes in sample density $\left(\right.$ e.g. $\left.\beta_{\text {new }}=\beta_{\text {nominal }} \rho_{\text {new }} / \rho_{\text {nominal }}\right)$. On the other hand, it is assumed that the variation in incident beam energy in typical experimental conditions is too small to have a significant effect on the refractive index. For example, changing the energy from 12.71 to $12.72 \mathrm{keV}$ changes $\beta$ and $\delta$ by only 0.3 and $0.2 \%$, respectively, for PTB 7 . $\beta$ and $\delta$ can be calculated using the refractive index calculator as discussed in the previous section (see Fig. 2).

The first purpose of our scattering tool is to demonstrate how scattering intensity depends on the experimental setup. The second purpose is to determine the uncertainty in the normalization factor given uncertainties in each of the experimental variables. Usually, the uncertainty in each variable can be approximated from instrumental specifications (such as profilometer accuracy) or from value ranges observed

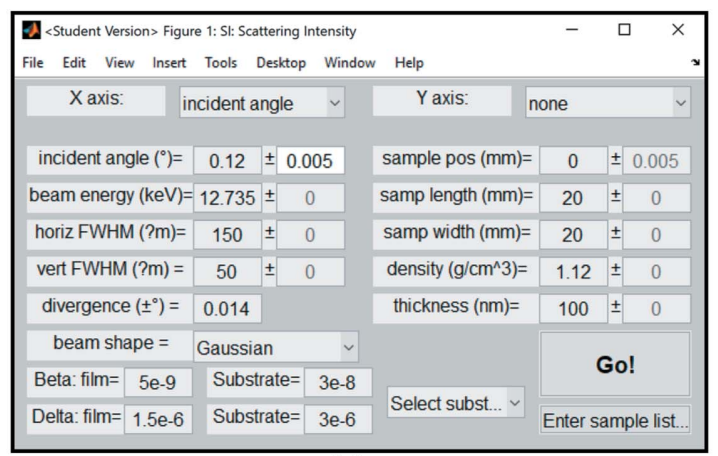

(a)

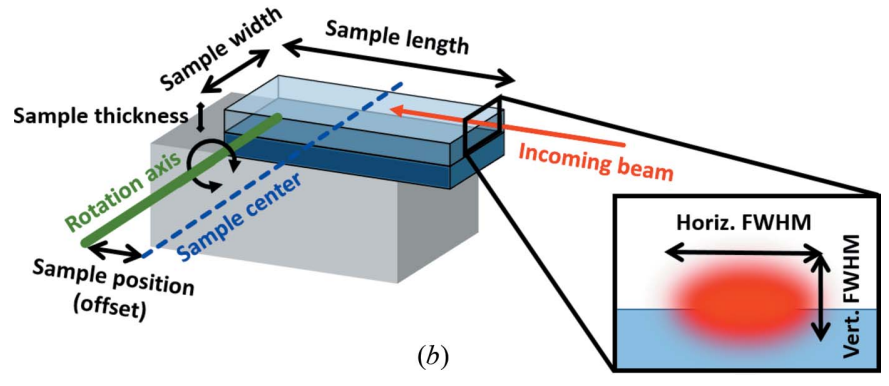

Figure 3

(a) User interface for intensity uncertainty calculation. The dropdown menus for $X$ axis and $Y$ axis both include entries for 'none', 'incident angle', 'horizontal beam width', 'vertical beam width', 'beam energy', 'sample position', 'sample length', 'sample width', 'sample density' and 'sample thickness'. The dropdown menu for beam shape includes 'Gaussian', 'Lorentzian' and 'Rectangular'. (b) Illustration showing variable definitions: the sample is shown in blue (substrate and film) and the sample stage is gray. in the literature (such as sample density range). Some considerations of the source of uncertainties for each variable are given in Table S2. Quantifying the propagation of individual uncertainties to an overall uncertainty in scattering intensity is not straightforward.

Our tool uses a Monte Carlo method to calculate expected overall uncertainties given uncertainties in up to five variables (Bevington \& Robinson, 2003; Koehler et al., 2009). The simulation is launched from the interface shown in Fig. 3 by selecting 'none' for both the $X$ and $Y$ axes. For this method, random numbers are generated for incident angle, sample position, sample length, density and thickness, following a Gaussian distribution. Each Gaussian distribution will be centered on the nominal value for the variable and have a standard deviation (std.dev.) set by the \pm box. The resultant scattering intensities are recorded in an array. The uncertainty is calculated from the set of scattering intensities using the formula

$$
\begin{aligned}
\operatorname{Uncertainty}(M) & =\left[\frac{\sum_{i=1}^{M}\left(I_{i}-\bar{I}\right)^{2}}{M-1}\right]^{1 / 2}\left(\frac{100 \%}{\bar{I}}\right) \\
& =\operatorname{std.dev.(I)}\left(\frac{100 \%}{\bar{I}}\right),
\end{aligned}
$$

where $I_{i}$ is the calculated scattering intensity for a single simulation, $\bar{I}$ is the average intensity value over the set of simulations (the expected value) and $M$ is the number of simulations. The uncertainty is calculated with respect to the average value in order to make it easily applicable to an arbitrary experimental intensity value. Monte Carlo simulations are added until the uncertainty value converges (Janssen, 2013). Convergence is determined when the slope and standard deviation of the last 1000 uncertainty measurements are less than 0.0001 and $0.01 \%$, respectively. In other words, convergence is reached when adding more simulations does not change the measured uncertainty systematically (slope) or randomly (standard deviation). Final uncertainties are taken as the average value of the last 1000 uncertainty measurements to minimize the effects of 'outlier' simulations. The user is prompted to enter a cut-off time limit, in seconds, to avoid excessive computation time.

\subsection{Calculation of $\boldsymbol{F}_{\text {footprint }}$}

In this section, we discuss the effect of changing beam footprint on scattering intensity. We use a straightforward simulation to calculate the beam footprint on the sample given the sample size and position, beam size and shape, and incident angle. The beam cross section is assumed to be ovalshaped and several beam intensity profile estimations are available: elliptical (Gaussian edges), elliptical (Lorentzian) and elliptical (sharp edges), and rectangular (sharp edges). Both horizontal and vertical beam FWHM can be defined in the GUI. Instructions for inserting a custom beam shape and profile are available in the user guide. The beam is projected onto a substrate with a given size and integrated within the sample region to give $F_{\text {footprint }}$, the fraction of the beam that 


\section{computer programs}

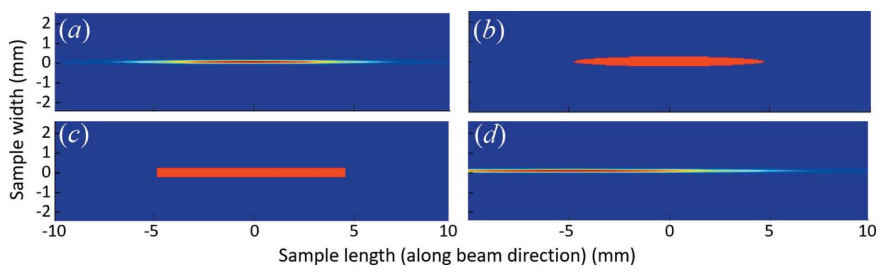

Figure 4

Examples of footprints of the beam on a $20 \times 5 \mathrm{~mm}$ substrate with varied beam types and geometries. (a) $150 \times 50 \mu \mathrm{m}$ (horizontal $\times$ vertical) elliptical (Gaussian) beam with an incident angle of $0.30^{\circ}$ and sample position of 0 (centered). (b) $500 \times 50 \mu \mathrm{m}$ elliptical (hard edges) beam, same conditions as (a). (c) $500 \times 50 \mu \mathrm{m}$ rectangular (hard edges) beam, same conditions as $(a)$. (d) $150 \times 50 \mu \mathrm{m}$ elliptical (Gaussian) beam with an incident angle of $0.18^{\circ}$ and a sample position of $5 \mathrm{~mm}$.

hits the sample. A few examples of beam footprints with various options selected are illustrated in Fig. 4: an elliptical (Gaussian edges) beam is shown in Fig. 4(a); an elliptical (hard edges) beam is shown in Fig. 4(b); and a rectangular (hard edges) beam is shown in Fig. 4(c). Fig. 4(d) shows the effect of introducing a sample position offset with an elliptical (Gaussian edges) beam.

When the sample is very large and/or the incident angle is large such as in Figs. 4(a) and 4(b), most of the beam will hit the sample and the fraction of the beam that is incident on the sample will be constant with changing parameters. However, at grazing angles, the beam will spread along the sample such that the beam spills over the sample surface, as in Fig. 4(c). In this example, the incident intensity will depend on sample length. In addition, the position of the sample relative to the beam center becomes important for certain configurations, as shown in Fig. 4(d). When the sample is aligned by hand it can be difficult to have a high degree of consistency in this variable, and the sample position may hence contribute to intensity uncertainty.

\subsection{Calculation of $\int_{0}^{T}|E(z)|^{2} d z$}

Next, we discuss the intensity of the beam within the sample (specifically within a thin film), which can be used to estimate the effective scattering volume. At grazing incidence, the electric field within the film has a large dependence on the incident beam angle (Chabinyc, 2010; Klockenkàmper et al., 1992; Müller-Buschbaum, 2014). The derivation of the electric field within a thin film, assuming negligible roughness and sharp interfaces, is shown in Appendix $A$. The electric field is calculated using the recursive Parratt formalism for a single layer on a substrate (Parratt, 1954; Boer, 1991; Dev et al., 2000). The calculation ignores the possibility of multiple diffraction events (Vineyard, 1982; Stepanov et al., 1998). The result, which is also derived in the work of Factor (1991) and Factor et al. (1993), is

$$
|E(z)|^{2}=\left|\left(1+r_{01}\right) \frac{\exp \left(i k_{1} z\right)+r_{12} \exp \left[i k_{1}(2 T-z)\right]}{1+r_{01} r_{12} \exp \left(i 2 k_{1} T\right)}\right|^{2},
$$

with

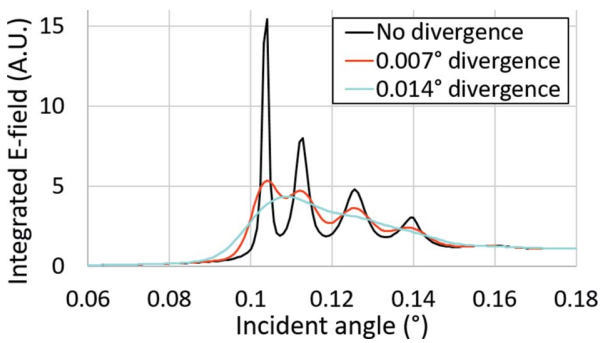

Figure 5

Integrated electric field for a $100 \mathrm{~nm}$-thick PTB7 film on silicon as a function of incident angle, with and without convolution by a Gaussian divergence with FWHM as indicated.

$$
r_{01}=\frac{k_{0}-k_{1}}{k_{0}+k_{1}} \quad \text { and } \quad r_{12}=\frac{k_{1}-k_{2}}{k_{1}+k_{2}}
$$

where $r_{01}$ and $r_{12}$ are the fractional Fresnel reflection coefficients at the top interface and at the film-substrate interface, respectively. The derivation of the expressions for $r_{01}$ and $r_{12}$ can for example be found in the work of Borne \& Wolfe (1985) and Als-Nielsen \& McMorrow (2011). $k_{0}, k_{1}$ and $k_{2}$ are the vertical components of the X-ray wavevectors incident on the film, inside the film and inside the substrate, respectively. The incident wavevector is $k_{0}=k \sin \theta_{0} \simeq k \theta_{0}$ for GIWAXS, where $k=\omega / c=2 \pi / \lambda$ and $\theta_{0}$ is the incident angle. This calculation was performed for horizontally or $s$-polarized X-rays (see Daillant \& Gibaud, 2009).

An expression for the wavevectors inside a stratified sample has been derived previously (Parratt, 1954; Factor, 1991; Borne \& Wolfe, 1985; Als-Nielsen \& McMorrow, 2011). We have included a derivation in Appendix $A$ for convenience (this assumes that the refractive index of the top medium, vacuum or inert gas, is unity). The wavevector for the $m$ th layer is

$$
\begin{aligned}
k_{m} & =k_{m, \operatorname{Re}}+i k_{m, \operatorname{Im}}, \\
k_{m, \operatorname{Re}}^{2} & =\frac{1}{2}\left\{\left[\left(k_{0}^{2}-2 k^{2} \delta_{m}\right)^{2}+4 k^{4} \beta_{m}^{2}\right]^{1 / 2}-2 k^{2} \delta_{m}+k_{0}^{2}\right\}, \\
k_{m, \operatorname{Im}}^{2} & =\frac{1}{2}\left\{\left[\left(k_{0}^{2}-2 k^{2} \delta_{m}\right)^{2}+4 k^{4} \beta_{m}^{2}\right]^{1 / 2}+2 k^{2} \delta_{m}-k_{0}^{2}\right\},
\end{aligned}
$$

where $\delta$ and $\beta$ are the real and imaginary components, respectively, of the refractive index $(n=1-\delta+i \beta)$. The subscript $m$ refers to the layer number with $1=$ film and $2=$ substrate.

Note that this calculation is carried out for the simplest case of a thin film, i.e. a single slab of constant electron density between the substrate and vacuum with ideally sharp interfaces with the substrate and vacuum (step function). This may be a significant limitation for some films. However, the electric field within more complex systems can also be calculated and we refer the reader to the literature (Stepanov et al., 1998; Gann et al., 2018).

Some results of the calculation of the dependence of the electric field on incident angle are shown in Fig. 5 and Fig. 16 in Appendix $A$. Interference of the incoming $\left[\exp \left(i k_{1} z\right)\right]$ and reflected $\left[\exp \left(-i k_{1} z\right)\right]$ beams results in oscillations in 
$\int_{0}^{T}|E(z)|^{2} \mathrm{~d} z$ versus incident angle. The slope of the integrated field plot in Fig. 5 can be very high near the oscillations, which means that a small error in incident angle alignment can result in a very large change in scattering intensity.

Notably, this calculation assumes an ideal incident beam with no divergence. In practice, X-ray sources have some beam divergence, which manifests as a spread in incident angles around the nominal angle. Accordingly, the effect of divergence must be added to the calculation of integrated electric field versus incident angle. In our program, we approximate the beam divergence as a Gaussian with FWHM corresponding to divergence angle and convolve this with the intensity versus angle curve (Fiorito et al., 2006). This effect is demonstrated in Fig. 5 with a divergence angle of $0.014^{\circ}$, which is typical for Stanford Synchrotron Radiation Lightsource beamline 11-3 (Rabedeau, 2006; Johnson, 2012), and also with a divergence angle of $0.007^{\circ}$. The convolution makes the oscillations in the $\int_{0}^{T}|E|^{2} \mathrm{~d} z$ versus incident angle curve less dramatic and therefore more forgiving towards incident angle misalignment; we note that the divergence also affects the preciseness achievable upon depth profiling a film via variation of incidence angles close to the critical angle.

Below the critical angle, the beam only penetrates the top $\sim 5-10 \mathrm{~nm}$ of film [as shown in Fig. 16(a)], rendering scattering at these low angles surface sensitive. The effective scattering volume is small and long exposure times may be needed. Above the critical angle, the penetration depth quickly rises to over $10 \mu \mathrm{m}$, which is well above the typical thickness of organic thin films. Thus, scattering at angles above the critical angle is bulk sensitive. The shape of the GIWAXS scattering intensity versus incident angle curve can be indicative of vertical segregation of scattering components of the film. This effect has been previously explored (Gann et al., 2018; Jiang et al., 2011; Renaud et al., 2009). We include instructions to change the integration limits of $\int|E(z)|^{2} \mathrm{~d} z$ (assuming that the film has a uniform density and refractive index) in the user guide: for example, the electric field intensity in only the top $5 \mathrm{~nm}$ can be examined. This approximates the results reported by Gann et al. (2018), but more in-depth stratification analysis requires a full implementation of the Parratt formalism (Parratt, 1954; Dev et al., 2000) and is beyond the scope of our toolbox.

In cases where only a qualitative comparison of scattering pattern is required (e.g. peak position determination and not peak intensity), and under the condition that the scattering intensity is low (as with polymers), the primary purpose of tuning the incidence angle is to maximize the scattering intensity. For this purpose, the best practice is to tune the incident angle to the film critical angle, which can result in a scattering intensity enhancement of $4 \times$ compared with higher angles. This is in contrast to cases where a quantitative comparison between scattering patterns of different samples is desired, where the incident angle should be tuned to be larger than the critical angle. Organic materials typically have critical angles of around $0.1^{\circ}$ at incident energies near $12.7 \mathrm{keV}$ [see Table S1: critical angle $\left.=(2 \delta)^{1 / 2} \mathrm{rad}\right]$. Due to the abrupt changes in scattering intensity for angles close to the critical angle, a small error in incident angle alignment can have a very large effect on scattering intensity. From Fig. 5, an angular uncertainty between 0.10 and $0.11^{\circ}$ (a change of just $\pm 0.005^{\circ}$ ) can lead to a $\pm 90 \%$ uncertainty in intensity with an ideal beam or $\pm 21 \%$ for a beam with a $0.014^{\circ}$ angular divergence. Thus, quantitative intensity comparisons, such as calculation of relative degree of crystallinity, require a higher incident angle where the intensity is more stable with change in angle.

\section{Usage example for scattering intensity normalization}

To demonstrate our scattering intensity tool, we present a calculation of a hypothetical sample set. We define our set to contain five samples which are all composed of neat PTB7 $\left(\mathrm{C}_{41} \mathrm{H}_{53} \mathrm{FO}_{4} \mathrm{~S}_{4}\right)$ films on silicon substrates. We assume measured thicknesses of $(80,80,100,120,120) \pm 5 \mathrm{~nm}$ and sample lengths of $(15,20,20,20,25) \pm 1 \mathrm{~mm}$, respectively. For all samples, we assume a density of $1.12 \pm 0.1 \mathrm{~g} \mathrm{~cm}^{-3}$, an incident angle of $0.140 \pm 0.005^{\circ}$, a $150 \times 50 \mu \mathrm{m}$ beam with an energy of $12.7 \mathrm{keV}$, and a $0.014^{\circ}$ beam divergence. Our goal is to correctly normalize and calculate uncertainties for this hypothetical data set. We assume that the exposure time and the incoming beam intensity $I_{0}$ are equal for all five samples.

The first step is to find normalization factors for the samples. We can get a general idea of how the normalization factor changes within this sample set by generating an $F_{\text {footprint }} \int_{0}^{T}|E(z)|^{2} \mathrm{~d} z$ intensity image with axes of thickness and length, as shown in Fig. 6(a). The result in Fig. 6(b) shows nonlinearity of the normalization factor with both variables, showing the importance of a robust normalization strategy over a simple sample dimension normalization.

We can take normalization factor values directly from the image for each of the five samples, but this can become time consuming when there are many samples or more than two (a)

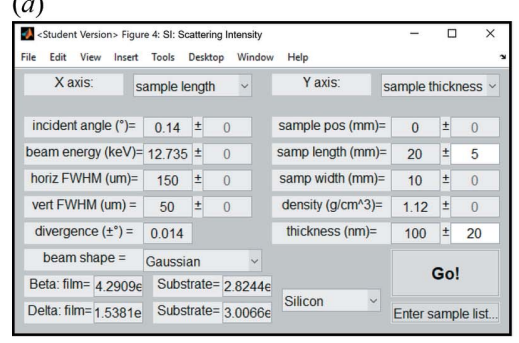

(b)

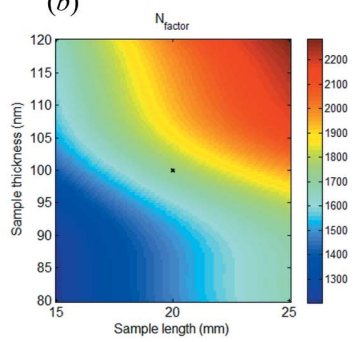

(c)

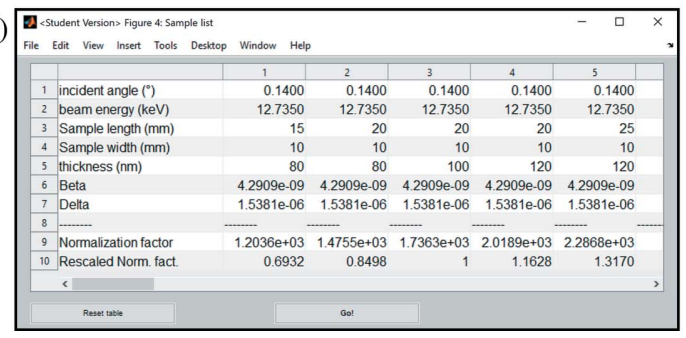

Figure 6

(a) Setup to examine change in scattering intensity for a sample set with length and thickness variation. (b) Result of $(a)$ : how the normalization factor changes with changing sample length and thickness. (c) Normalization factors calculated for a sample set within the parameters set in $(a)$. 
variables that change between samples. For this reason we include an additional tool that calculates the normalization factor for specific samples rather than for a range of values. This can be accessed via the 'Enter sample list...' button shown in Fig. 6(a).

The sample list tool interface is shown in Fig. 6(c). It consists of a table where each column represents a single sample and the first seven rows provide input variables for each sample. Parameters related to beam dimensions (horizontal/vertical FWHM, divergence and beam shape) and sample offset are assumed to be constant for the sample set and are taken from the main GUI. Once the variables are entered, the 'Go!' button calculates the normalization factors and reports them in row 9. It also gives a rescaled set of normalization factors in row 10 that are scaled to have a median value of 1 (this will change between sample sets). These rescaled normalization factors can be used to avoid drastic changes to overall intensity which could require modifying existing data processing methods (for example, changing the limits of peak fitting).

Next we consider the uncertainty in the normalization factors for the films in this set [lengths and thicknesses shown in Fig. 6(c)], starting with the film with nominal thickness $100 \mathrm{~nm}$ and substrate length $20 \mathrm{~mm}$. The sources of uncertainty are all sample dependent, and include incident angle $\left( \pm 0.005^{\circ}\right)$, sample position $( \pm 3 \mathrm{~mm})$, sample length $( \pm 1 \mathrm{~mm})$, density $\left( \pm 0.1 \mathrm{~g} \mathrm{~cm}^{-3}\right)$ and thickness $( \pm 5 \mathrm{~nm})$. The setup for launching a Monte Carlo error propagation calculation for this sample is shown in Fig. S1 $(a)$, and the calculated convergence in uncertainty and spread in intensities are shown in Figs. S1(b) and $\mathrm{S} 1(c)$. The simulation shown here reached convergence with 7310 iterations (i.e. 7310 simulated samples). The histogram of normalization factor intensities had a standard deviation of $17.4 \%$. Repeating this calculation for all five samples gives standard deviation uncertainties of $(19.2,18.9$, $17.4,17.4,17.3) \%$. Due to the random nature of Monte Carlo simulations, there may be slight differences in converged uncertainties between consecutive calculations (Koehler et al., 2009). For this sample set, we have observed this difference to be on the order of $0.4 \%$ (e.g. between 19.1 and $19.5 \%$ ). The

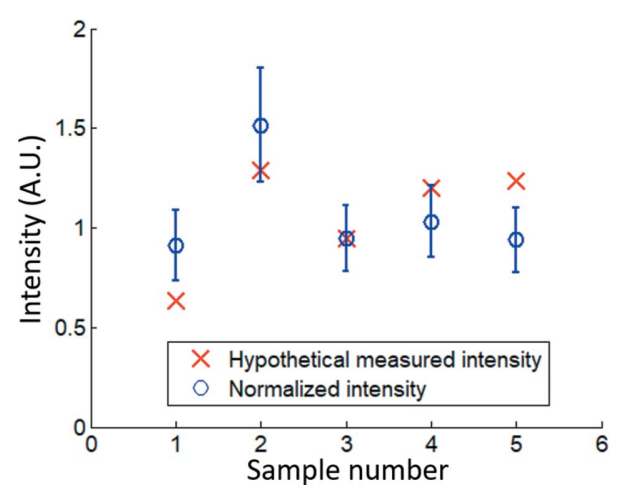

Figure 7

Hypothetical peak intensity data collected from the sample set in Fig. 6(c): raw data (red crosses) and normalized intensity with calculated uncertainty shown as error bars (blue). number of iterations required for convergence was between 7300 and 11200.

To illustrate the importance of taking a normalization factor into account, we provide a hypothetical experiment, as shown in Fig. 7. The hypothetical scattering data, shown as red crosses, vary across the sample set: samples 2, 4 and 5 have approximately the same measured intensity, sample 3 is slightly lower, and sample 1 is even lower. Once the data are normalized (blue circles), however, we can see that the differences between samples $1,3,4$ and 5 are within the uncertainty, while the intensity of sample 2 is significantly higher. Thus, appropriate normalization changes the conclusions drawn from this hypothetical sample set significantly.

\section{Calculation of refractive shift and reflected peak position}

$\mathrm{X}$-ray scattering peak positions are slightly shifted due to refraction at the interface between the sample and the atmosphere (Toney \& Brennan, 1989). These effects change the vertical angle of scattering, while leaving the horizontal angle unchanged. Applying a correction to the observed scattering pattern (peak positions) to account for refractive shift is important to accurately retrieve $d$ spacing and crystal structure. It is especially important to consider refractive shift when comparing films with different compositions, such as blend ratio titrations: changes to average refractive index will change the observed peak position even when $d$ spacing is constant (Savikhin, Babics et al., 2018). Here, we assess the magnitude of this refractive effect in organic materials.

In this derivation and in our script we have assumed that the $Q_{x y}-Q_{z}$ map is based on the $Q_{z}$ center being at the direct beam (as opposed to the horizon). This approximation ignores the standing wavefield of Section 4.2 because this estimate is widely used in the literature. We note that the relative changes in peak positions (e.g. differences between red and blue curves in Fig. 10) are not significantly affected by this approximation. Research into accurate unwarping of scattering images is ongoing (Liu \& Yager, 2018).

The relationships between vectors and angles in a scattering experiment are shown graphically in Fig. 8. The incident beam, $k_{0}$ (at an angle of $\theta_{0}$ ), is refracted to $k_{1}$ (at an angle of $\theta_{1}$ ), as shown in Fig. 8(a). Both $k_{0}$ and $k_{1}$ are in the $X-Z$ plane (as marked in Fig. 8). $k_{1}$ is scattered to $k_{1 \mathrm{~s}}$, which has components along $X, Y$ and $Z$. The scattered beam can be expressed in terms of a rotation angle in the $X-Y$ plane $\left(\theta_{1 \mathrm{~s}, X Y}\right)$ and a vertical angle $\left(\theta_{1 \mathrm{~s}, Z}\right)$ [see Fig. $\left.8(b)\right]$. The scattered beam refracts on exiting the film to become $k_{0 \mathrm{~s}}$, which has the same in-plane angle $\theta_{0 \mathrm{~s}, X Y}=\theta_{1 \mathrm{~s}, X Y}$ and a refracted vertical angle $\left(\theta_{0 \mathrm{~s}, Z}\right)$ [see Fig. 8(a)].

In previous work (Toney \& Brennan, 1989), a grazingincidence out-of-plane measurement was considered in establishing the magnitude of the refractive shift. Only scattering in the $X-Z$ plane $\left(\theta_{1 \mathrm{~s}, X Y}=0\right)$ was measured in this experiment. The exit angle in this type of experiment is usually quite large, so refraction of the exit beam has a small effect 
and can be ignored $\left(k_{0 \mathrm{~s}} \simeq k_{1 \mathrm{~s}}\right)$. Thus, Toney \& Brennan (1989) obtained

$$
\begin{aligned}
\Delta 2 \theta & =2 \theta_{0}-2 \theta_{1}=\left(\theta_{0 \mathrm{~s}}+\theta_{0}\right)-\left(\theta_{1 \mathrm{~s}}+\theta_{1}\right) \simeq \theta_{0}-\theta_{1} \\
& \simeq \theta_{0}-\frac{1}{2^{1 / 2}}\left\{\left[\left(\theta_{0}^{2}-2 \delta\right)^{2}+4 \beta^{2}\right]^{1 / 2}-2 \delta+\theta_{0}^{2}\right\}^{1 / 2},
\end{aligned}
$$

where $2 \theta_{0}$ is the observed scattering angle and $2 \theta_{1}$ is twice the Bragg angle (without refraction). This equation arises from the expression for the real component of the wavevector given in Section 4.2, using the reasonable assumption that the imaginary component is negligible compared with the real component $\left(k_{1} \simeq k_{1, \mathrm{Re}}\right)$.

A similar method can be used for 2D scattering. In this case, the exit beam may have small vertical angles (at polar angles close to the horizon), so the refraction of the exit beam can no longer be ignored. Refraction only affects the vertical scattering angle (the rotation angle is unchanged; $\theta_{1 \mathrm{~s}, X Y}=\theta_{0 \mathrm{~s}, X Y}=$ $\left.\theta_{\mathrm{s}, X Y}\right)$. Thus, we must develop an expression to extract the vertical exit angle $\theta_{1 \mathrm{~s}, Z}$ from the scattering angle $2 \theta_{1}$ and the polar angle $\chi_{1}$ (defined such that $\tan \chi_{1}=Q_{z} / Q_{x v}$ ). This involves a straightforward but nontrivial calculation. We show this calculation, along with the full procedure for converting $Q_{x y}$ and $Q_{z}$ to polar angle and $d$ spacing (and vice versa), in Appendix $B$. Another approach for finding this correction, derived with a focus on small-angle X-ray scattering/smallangle neutron scattering, can be found in the work of Busch et al. (2011).

In addition to refractive shift, GIWAXS data of well ordered organic materials may display a 'double-peak' effect,
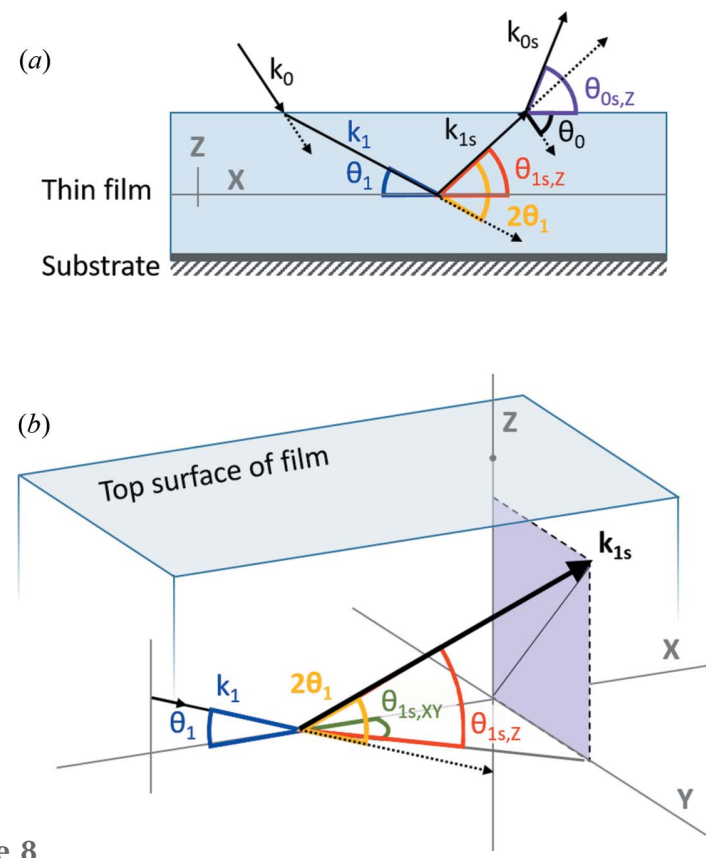

Figure 8

Setup for calculating refractive shift as a function of $2 \theta_{1}$ and $\chi_{1}$. (a) Side view, showing the two refractive shifts: $k_{0} \rightarrow k_{1}$ and $k_{1 \mathrm{~s}} \rightarrow k_{0 \mathrm{~s}}$ (angles have been exaggerated for clarity), ignoring in-plane rotation $\left(\theta_{\mathrm{s}, X Y}\right)$. (b) $3 \mathrm{D}$ view of scattering within the film, showing rotational and vertical components of the scattering angle. especially in the out-of-plane direction, as shown by Resel et al. (2016) and in Fig. 9(a). This additional peak is due to reflection of the scattered beam off the substrate or scattering of the reflected beam, as shown in Figs. $9(c)$ and $9(d)$. It can be observed from Fig. 9(c) that the vertical exit angle of a beam that enters the film, scatters and then reflects will be $\theta_{1 \mathrm{~s}, Z}+2 \theta_{1}$ for incident angles greater than the critical angle. The beam shown in Fig. $9(d)$, which passes through the film, reflects and then scatters, will have the same exit angle of $\theta_{1 \mathrm{~s}, Z}+2 \theta_{1}$. The vertical component of the reflected peak will also be refracted as it exits the film. The horizontal angle of the reflected peak is the same as that of the scattered beam $\left(\theta_{\mathrm{s}, X Y}\right)$ because the horizontal angle is unchanged with reflection. When comparing peak intensities, it is most accurate to sum the intensities of the first and second peak.

An additional peak or shoulder can also arise when two or more similar $d$ spacings are present in a material (Savikhin, Jagadamma et al., 2018). Accordingly, it is imperative to distinguish these two effects. The position and intensity of the reflected peak change more dramatically with incident angle than the position and intensity of directly scattered peaks. The highest intensity of the reflected peak occurs when the incident angle is between the critical angle of the film and the critical angle of the substrate. An example of an observed reflected peak for a well ordered small molecule is shown in Fig. $9(a)$. The film critical angle is calculated to be $0.10^{\circ}$. The reflected peak overlaps with the main peak (which is at $Q_{z} \simeq$ $0.33 \AA^{-1}$ ) at an incident angle of $0.11^{\circ}$ but moves to higher $Q_{z}$
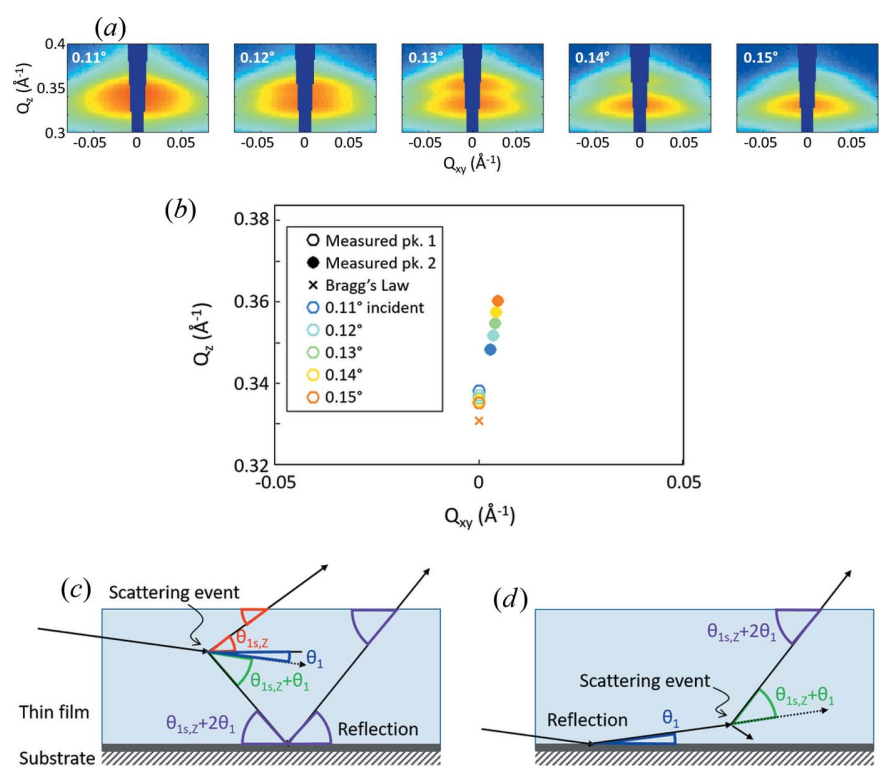

Figure 9

(a) Data demonstrating the double-peak effect for the small molecule DR3TBDTT (Liang et al., 2018) with $12.7 \mathrm{keV}$ incident energy, sampledetector distance $250 \mathrm{~mm}$, incident angle as indicated. (b) Simulation results for the system in $(a)$. The peak position corresponding to Bragg's law is shown as a cross; the observed peaks for various incident angles are shown as open circles and dots. Each color corresponds to a different incident angle as marked. (c) Ray tracing of the reflected peak effect with a beam that is first scattered and then reflected. $(d)$ Reflected peak effect when the direct beam is reflected and then scattered. 
as the incident angle is increased, becoming especially prominent at 0.13 and $0.14^{\circ}$. The intensity of the reflection peak falls off at incident angles above $0.13^{\circ}$, and by an incident angle of $0.15^{\circ}$ this peak is barely visible. Another example showing the movement of a reflected peak with incident angle is reported by Resel et al. (2016).

We have written a MATLAB script that performs both the refractive shift and the reflected peak calculations for a user, given the interface shown in Fig. 10(a). The user is first required to enter the beam energy, incident angle, and $\beta$ and $\delta$ values, which may be calculated using the refractive index tool described in Section 3. The GUI toggles between starting with experimentally observed $\left(Q_{x y}, Q_{z}\right)$ positions and calculating $\left(\chi_{1}, 2 \theta_{1}, d\right.$ spacing) (the procedure described above) or starting with $\left(\chi_{1}, d\right.$ spacing) and calculating $\left(Q_{x y}, Q_{z}\right)$ (the reverse procedure). The user can enter values manually or import $\left(Q_{x y}, Q_{z}\right)$ positions from a file, such as the peak position file generated by our peak finder tool (see Section 7). For convenience, if the user leaves any input cells blank, they will be filled in by duplicating the values in the previous row. Once all the desired peaks are specified, the 'Go!' button launches the calculation to fill in the table.

In addition to the table, a $Q_{x y}-Q_{z}$ pattern with the 'measured' and 'Bragg's law' peak positions is displayed [Fig. 9(b)]. The 'measured' positions refer to what is experimentally observed (for both the directly scattering and reflected peaks) while the 'Bragg's law' position is corrected for refraction. We used our tool to predict the positions of the measured peaks for the film in Fig. 9(a). Our results, shown in Fig. $9(b)$, are consistent with the experimentally observed peak positions. We also used a similar procedure to predict the peak positions observed by Resel et al. (2016) and found good consistency, as shown in Appendix $B$.

Some results of the calculation are shown in Figs. 10(b) and 10(c) for neat PTB7. Increasing the $d$ spacing does not

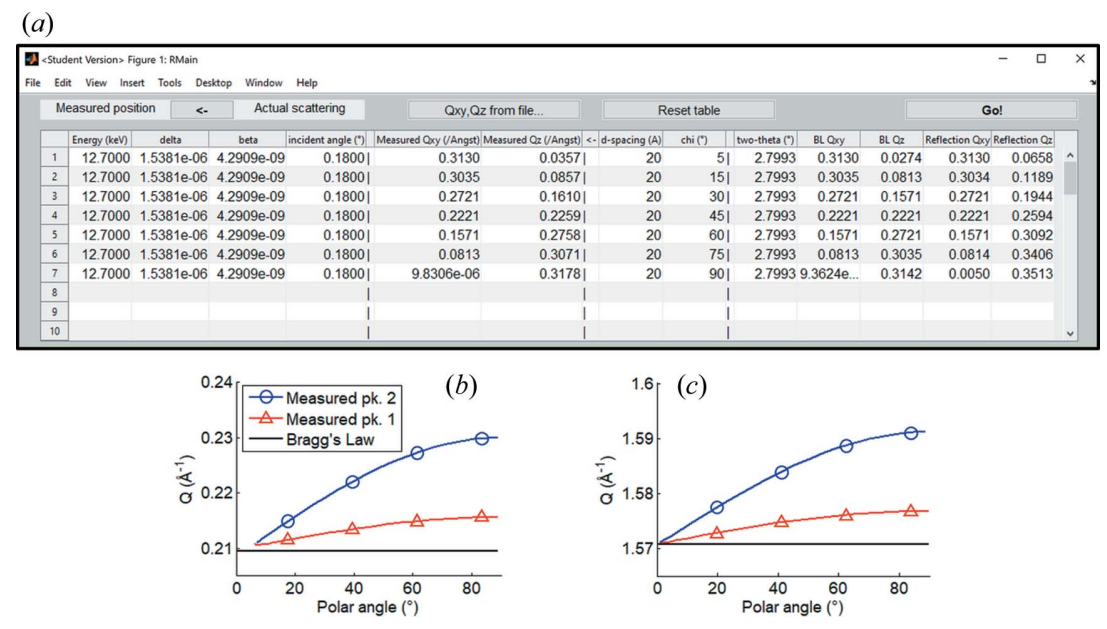

Figure 10

(a) Example usage of the refractive shift calculator tool, set up to calculate measured positions of seven peaks at a $d$ spacing of $20 \AA$ and various polar angles. $(b)$ Results of the refractive shift calculation for PTB7 if $d=30 \AA$ and incident angle $=0.12^{\circ}$. (c) Results for $d=$ $4 \AA$ and incident angle $=0.12^{\circ}$. A polar angle of $0^{\circ}$ represents in-plane scattering (along $Q_{x y}$ ), while a polar angle of $90^{\circ}$ represents out-of-plane scattering (along $Q_{z}$ ). significantly change the magnitude of $\Delta Q$ for either refraction or reflection. In this example, for both $d=30 \AA$ [Fig. 10(b)] and $d=4 \AA$ [Fig. 10(c)], refraction changes the measured outof-plane $Q$ position of the directly scattered peak by $0.006 \AA^{-1}$ relative to the Bragg's law position. The separation between the directly scattered and reflected peaks is $0.014 \AA^{-1}$ for both $d$ spacings. Therefore the refractive shift has a smaller fractional effect on measured $d$ spacing at higher $Q$. Meanwhile, the magnitude of the refractive shift decreases at higher incident angles.

The angular (or $Q$ ) resolution in most GIWAXS experiments is limited by the footprint projected onto the detector at a given scattering angle (as shown in Section 4.2), leading to geometric broadening of all features (Smilgies, 2009). For a rectangular beam this corresponds to $B_{\text {geo }}=(w \tan 2 \theta) / L$, where $B_{\text {geo }}$ is the broadening factor, $L$ is the sample-todetector distance, $w$ is the footprint and $2 \theta$ is the Bragg scattering angle. In practice, one can limit this effect by using smaller samples (effectively reducing the footprint to the sample size), higher X-ray energies (yielding smaller $2 \theta$ ) or Soller collimators (Als-Nielsen et al., 1994). For mathematical peak shape analysis, or peak splitting analysis, the intrinsic peak profiles then need to be convoluted with the experimental resolution function (Steinrück et al., 2014), which also includes energy bandwidth and beam divergence (Smilgies, 2009) (often negligible in GIWAXS due to geometric broadening).

\section{Indexing software}

Small-molecule-based films tend to scatter more distinctly than polymers, since they are often better ordered, sometimes generating scattering patterns with many well resolved peaks. By indexing each diffraction peak, it becomes possible to establish a real-space unit cell and to start comparing differences in molecular packing between films (Servet et al., 1993; Akimichi et al., 1993; Sherman et al., 2015; Mannsfeld et al., 2011). This process can be greatly aided by prior knowledge of expected packing distances from previous literature or simulation (Nabok et al., 2007). The orientation of the molecule within the unit cell can be calculated from the relative intensities of the scattering peaks, which are governed by the molecular structure factor along with several correction terms (Mannsfeld et al., 2011). For organic materials it can be difficult to measure peak intensities with a high degree of accuracy due to the small scattering signal, broad peaks due to disorder and overlap with scattering from other sources (especially in blend films). It is sometimes possible to prepare a single crystal of an organic small molecule which will alleviate these problems (Chang et al., 2012; Kim, Liu et al., 2012). However, not all materials can be coaxed into a highly crystalline form, 
and single crystals must be produced using different processing than that used for thin films which may favor a different polymorph (Sherman et al., 2015; Fritz et al., 2004). Therefore, we developed a software package to find a unit cell from a GIWAXS pattern with constraints designed specifically to cater to small-molecule thin films that gives insight into the molecular packing. We require some user-specified restrictions in order to minimize computational time and to select a unit cell where the primary lattice directions are more likely to correspond to molecular dimensions (i.e. the $\pi-\pi$ stacking spacing). This assumes a lamellar-type packing structure in which the unit cell corresponds to a single molecule. The software may be applied to obtain larger unit cells, such as those seen with a herringbone configuration (Sherman et al., 2015), but this alone will not show how molecules pack within the unit cell.

Thus, our goal is to obtain a unit cell in which the primary crystallographic directions align with the dimensions of the small molecule, with an assumption of lamellar packing. This unit cell will give us information on the intermolecular distances, which determine the ease of charge hopping and Förster energy transfer (Olivier et al., 2006; Kippelen \& Brédas, 2009). We can also extract slip-stacking (the offsets labeled 's' in Fig. 14 below), which has been shown to affect triplet-triplet interactions in an oscillatory fashion: small changes in slip-stack distance can dramatically change the triplet-triplet coupling (Hartzler et al., 2018). There is (a)

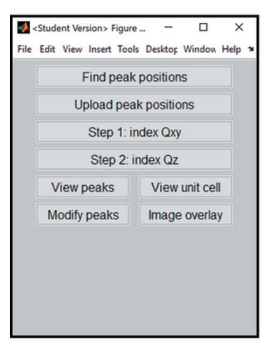

(d)

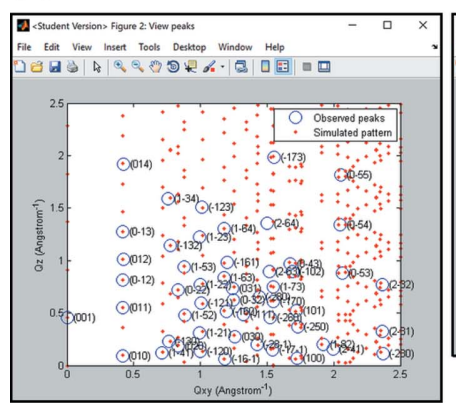

(b)

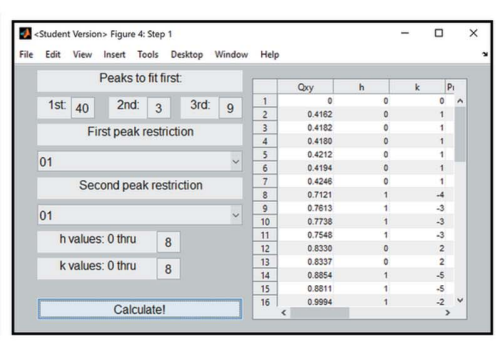

(e)

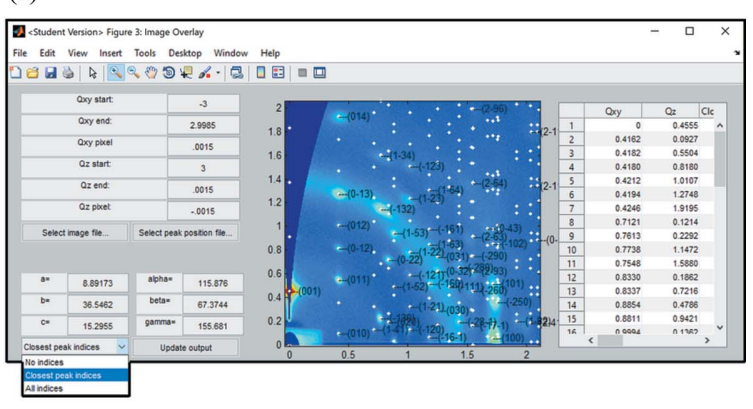

Figure 11

A selection of screenshots of interfaces used for indexing SM1. Additional screenshots are given in the user guide along with a step-by-step procedure. (a) Main interface for the peak indexing toolkit. (b) The interface for 'Step 1' of fitting, populated with fit results. The table has columns of $Q_{x y}, h, k$, Predicted $Q_{x y}$, Error $\left(\Delta Q_{x y}\right)$, and a single row with $a_{\|}^{*}, b_{\|}^{*}$ and $\gamma_{\|}^{*}$. This GUI is similar in format to the GUI for 'Step 2'. (c) Main interface after indexing is completed: updated with results. $(d)$ Result of 'View peaks' button. This is one of several tools that can be used to view or modify the results. $(e)$ Result of 'Image overlay' button, after loading an image file. Unit-cell parameters may be changed manually. The user can choose to display all peak indices, only peak indices closest to measured peak positions or no peak indices, in addition to the predicted scattering pattern (white dots). evidence that the use of triplets may improve OPV efficiency due to their longer lifetime (Köhler \& Bässler, 2009), and even enable solar cell devices with internal quantum efficiencies approaching $200 \%$ utilizing singlet-triplet exciton fission (Congreve et al., 2013). Finally, the thin-film structures obtained from GIWAXS can be compared with single-crystal counterparts (if available) using a software package such as DPC (Hailey et al., 2014) or SUNBIM (Siliqi et al., 2016).

Small molecules and polymers in a lamellar packing structure have been widely observed to have periodicity along the alkyl side-chain direction (repeat length in the range of 10$30 \AA$, depending on molecular structure), the $\pi-\pi$ stacking direction (3.5-4.0 $\AA$ ) and the direction along the backbone (Liu et al., 2013). Identifying which peaks correspond to the primary molecular directions is not trivial, as peak intensity depends on a variety of factors. However, it is reasonable to assume that the alkyl and $\pi-\pi$ stacking directions give rise to the strongest scattering peaks because these directions have the highest electron-density differential and the most extended (coherent) periodicity. There may be a high electron-density differential in the direction along the backbone, but backbone torsion may give rise to high disorder in this direction which decreases peak intensity. In addition to qualitative peak intensity, the position of a peak can give a clue about its origin. It is often assumed that the primary axes of the molecule (alkyl, $\pi-\pi$ and backbone) are approximately normal to each other, and the $d$ spacings given by $d=2 \pi / Q$ should be appropriate for the molecular dimensions.

The main interface of our tool is shown in Fig. 11(a). The first function (using the 'Find peak positions' button) is utilized to accurately extract peak positions from $Q_{x y}-Q_{z}$ patterns. Our custom peak-fitting script allows the user to load an image file, outline each peak location and fit each peak to a $2 \mathrm{D}$ oval-shaped pseudo-Voigt function on a planar background (following the form $C_{1} Q_{x y}+C_{2} Q_{z}+C_{3}$, where $C_{1,2,3}$ are fitted constants). For additional accuracy, peak positions may be adjusted using the refraction tool discussed in Section 6. The average peak width and intensity are also reported. These are not used in the indexing process by default but can be added as weighting factors following the minor modification described in the user guide. Alternatively, peak positions can be loaded from a text file using the 'Upload peak positions' button.

Once peak positions are established, 'Step 1' of the fitting routine determines preliminary $(h k)$ indices for each peak's $Q_{x y}$ position. The interface for 
this tool is shown in Fig. 11(b). We achieve this by solving the following equation, which is reproduced from the work of Merlo et al. (2005) for convenience:

$$
Q_{x y}^{2}=h^{2}\left(a_{\|}^{*}\right)^{2}+k^{2}\left(b_{\|}^{*}\right)^{2}+2 a_{\|}^{*} b_{\|}^{*} h k \cos \left(\gamma_{\|}^{*}\right),
$$

where $Q_{x y}$ is the experimentally measured horizontal peak position, $h$ and $k$ are the first two indices, $a_{\|}^{*}$ is the component of $a^{*}$ parallel to the substrate, $b_{\|}^{*}$ is the parallel component of $b^{*}$, and $\gamma_{\|}^{*}$ is the angle between the vectors $a_{\|}^{*}$ and $b_{\|}^{*} \cdot a^{*}$ and $b^{*}$ are the reciprocal-lattice vectors of the unit cell. The user specifies three peaks $\left(Q_{x y 1}, Q_{x y 2}, Q_{x y 3}\right)$ to be used for an initial guess. The program cycles through each possible $(h k)$ combination for these three peaks for values up to a userspecified maximum (for example, $-8 \leq h \leq 8$ and $-8 \leq k \leq 8$ ), and solves the following system of equations:

$$
\left(\begin{array}{l}
Q_{x y 1}^{2} \\
Q_{x y 2}^{2} \\
Q_{x y 3}^{2}
\end{array}\right)=\left(\begin{array}{lll}
h_{1}^{2} & k_{1}^{2} & h_{1} k_{1} \\
h_{2}^{2} & k_{2}^{2} & h_{2} k_{2} \\
h_{3}^{2} & k_{3}^{2} & h_{3} k_{3}
\end{array}\right)\left[\begin{array}{c}
\left(a_{\|}^{*}\right)^{2} \\
\left(b_{\|}^{*}\right)^{2} \\
2 a_{\|}^{*} b_{\|}^{*} \cos \left(\gamma_{\|}^{*}\right)
\end{array}\right] .
$$

The remaining peaks are assigned $(h k)$ values based on proximity (minimizing their $\Delta Q_{x y}$ ). The summed root-meansquare peak position error of all peaks $\left(\Delta Q_{x y}\right)$ is used to select the best set of values for $\left(h_{1}, k_{1}, h_{2}, k_{2}, h_{3}, k_{3}\right)$. Then $a_{\|}^{*}, b_{\|}^{*}$ and $\gamma_{\|}^{*}$ are refined to minimize the total peak position error.

In addition to setting an upper limit to $h$ and $k$ for all peaks, the user can set a further constraint on the $(h k)$ values that are allowed for the first two peaks $\left(h_{1}, k_{1}, h_{2}, k_{2}\right)$ based on expectations from the molecular size and knowledge from similar packing considerations. Typically, strong peaks are chosen that correspond to expected directions along the molecule. This encourages the software to report a unit-cell structure containing a single molecule (the smallest possible repeat volume) rather than multiple molecules: for example, by restricting the peak with the lowest observed $Q_{x y}$ to $(h k)=$ (01) rather than (02) or (03). This also allows the user to align molecular directions with primary crystallographic directions, which can make it easier to understand how a molecule orients within the unit cell. For example, a bright peak around $Q=$ $1.7 \AA^{-1}$ gives the appropriate $d$ spacing for $\pi-\pi$ stacking in a lamellar structure, and forcing this peak to have $(h k)=(01)$ means that one of the unit-cell axes will correspond to the $\pi-\pi$ stacking direction. Examples of how to choose appropriate constraints for a real system are given in Section 8 .

The second indexing step uses a similar approach to solve the following equation [also reproduced from Merlo et al. (2005)]:

$$
Q_{z}=h a_{\perp}^{*}+k b_{\perp}^{*}+\ell c^{*},
$$

where $Q_{z}$ is the experimentally measured vertical peak position, $(h k \ell)$ are the indices, $a_{\perp}^{*}$ and $b_{\perp}^{*}$ are the components of $a^{*}$ and $b^{*}$ perpendicular to the substrate, and $c^{*}$ is the out-ofplane reciprocal-lattice vector. An initial guess for $c^{*}$ is required (see next paragraph). The program runs through a series of guesses for $\left(\ell_{1}, \ell_{2}\right)$ to solve a set of two equations for $a_{\perp}^{*}$ and $b_{\perp}^{*}$ given two peaks specified by the user:

$$
\left(\begin{array}{c}
Q_{z, 1}-\ell_{1} c^{*} \\
Q_{z, 2}-\ell_{2} c^{*}
\end{array}\right)=\left(\begin{array}{ll}
h_{1} & k_{1} \\
h_{2} & k_{2}
\end{array}\right)\left(\begin{array}{l}
a_{\perp}^{*} \\
b_{\perp}^{*}
\end{array}\right) .
$$

These parameters are combined with the parameters obtained in the first indexing step to create a complete unit cell, which is used to generate a scattering pattern. The solution with the smallest total error, calculated as the sum of the Cartesian distances between each experimental peak and the closest generated peak, is chosen. The $a_{\perp}^{*}, b_{\perp}^{*}$ and $c^{*}$ values which lead to the lowest total error are then refined to further minimize error.

There are several methods to select a reasonable initial guess for $c^{*}$. In edge-on (or face-on) materials, there is usually a distinct out-of-plane alkyl peak (or $\pi-\pi$ stacking peak) that corresponds to the (001) index, and thus its $Q_{z}$ position is equal to $c^{*}$. The region along the $Q_{z}$ axis is inaccessible in grazing incidence, but ( $00 \ell)$ peak positions can often be estimated due to spread in the polar angle of these peaks. If not, the peaks can be measured using a specular scan. $c^{*}$ will also correspond to the distance $\Delta Q_{z}$ between peaks with identical $(h k)$ values $\left[\right.$ e.g. $\left.Q_{z(121)}-Q_{z(120)}=c^{*}\right]$, though a sign change in

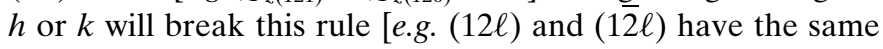
$Q_{x y}$ position, but their peaks may be separated by $\left.\Delta Q_{z} \neq c^{*}\right]$. A visual assessment of the scattering pattern can reveal a commonly repeated $\Delta Q_{z}$ value corresponding to $c^{*}$ or a multiple of $c^{*}$.

We have included several tools to inspect the results. Fit parameters are converted to real-space $a, b, c$ (unit-cell edge lengths), $\alpha, \beta, \gamma$ (unit-cell angles), and volume using the equations given by Merlo et al. (2005) and Simbrunner et al. (2018), and these are displayed in the main interface [see Fig. 11(c)]. The user can inspect generated peak positions from the unit cell overlaid with the experimentally defined peak positions [as shown in Fig. 11(d)]. 3D and 2D drawings of the real-space unit cell are also available. Furthermore, a tool is included to manually change each of six unit-cell parameters and inspect the corresponding scattering pattern in real time, and another tool can overlay the results on the experimental image [see Fig. 11(e)].

Organic thin films sometimes contain multiple polymorphs that result in more than one set of crystalline peaks. This makes it difficult to accurately assign crystal structure. Encouraging a material to convert to a single-crystal structure through a processing condition such as annealing above the melting temperature can help isolate peaks from different structures (Chang et al., 2012; Kim, Liu et al., 2012). Then, peaks in the original film that are not present in the annealed film can be assigned to a second polymorph. Different polymorphs often have different $c^{*}$ values, so an inspection of $\Delta Q_{z}$ can also help separate peaks from multiple polymorphs.

\section{Usage example of indexing software}

We demonstrate the use of our indexing tool by evaluating the crystal structure of two small-molecule donors, SM1 and SM2, that are based on the backbone benzo[1,2- $b: 4,5-b]$ dithiophenepyrido[3,4-b]-pyrazine $\left[\mathrm{BDT}\left(\mathrm{PPTh}_{2}\right)_{2}\right]$ and were studied in 
depth by Alqahtani et al. (2018) [also studied by Wolf et al. (2016), where they are referred to as SM3 and SM4, respectively]. These two molecules share a backbone but differ in the side chains attached to the BDT unit: SM1 has a 2-ethylhexyl side chain attached through an ether structure while SM2 has a 2-(2-ethylhexyl)3-hexylthiophene structure. Thus, SM1 has two side chains with weights of $112 \mathrm{~g} \mathrm{~mol}^{-1}$ each (eight carbon atoms, one oxygen atom) and SM2 has two side chains with weights of $248 \mathrm{~g} \mathrm{~mol}^{-1}$ each (18 carbon atoms, one sulfur atom). The difference in side chain results in substantial differences in GIWAXS patterns, indicating different packing structures. While the neat films are more disordered, both small molecules become highly crystalline when cast with the solvent additive diiodooctane (DIO) and blended with $\mathrm{PC}_{71} \mathrm{BM}$, allowing accurate peak indexing. We present the scattering patterns of the SM1: $\mathrm{PC}_{71} \mathrm{BM}$ blend in Figs. 12(a) and $12(b) ; \mathrm{SM} 2: \mathrm{PC}_{71} \mathrm{BM}$ in Figs. $13(a)$ and $13(c)$; and neat SM2 in Fig. 13(b).

For SM1, we harvested 53 peaks to use in indexing the crystal pattern. These are shown with white crosses in Fig. 12(a). Visual inspection of Fig. 12(a) reveals high-intensity peaks in the out-of-plane direction $\left(Q_{z}=0.46 \AA^{-1}\right)$ and inplane direction $\left(Q_{x y}=1.72 \AA^{-1}\right)$. The $d$ spacings corresponding to these peaks fall in the range expected for the alkyl and $\pi-\pi$ stacking distances, respectively, so we start with the assumption that these peaks can be assigned to the (001) and (100) indices. The positions of these peaks point to an edge-on structure.

Step 1 requires us to select three peaks for an initial calculation. These should have nonzero $Q_{x y}$ values that are not multiples of each other. For peak 1 , we select the probable $\pi-$ $\pi$ stacking peak at $\left(Q_{x y}, Q_{z}\right)=(1.724,0.066) \AA^{-1}$ and restrict it

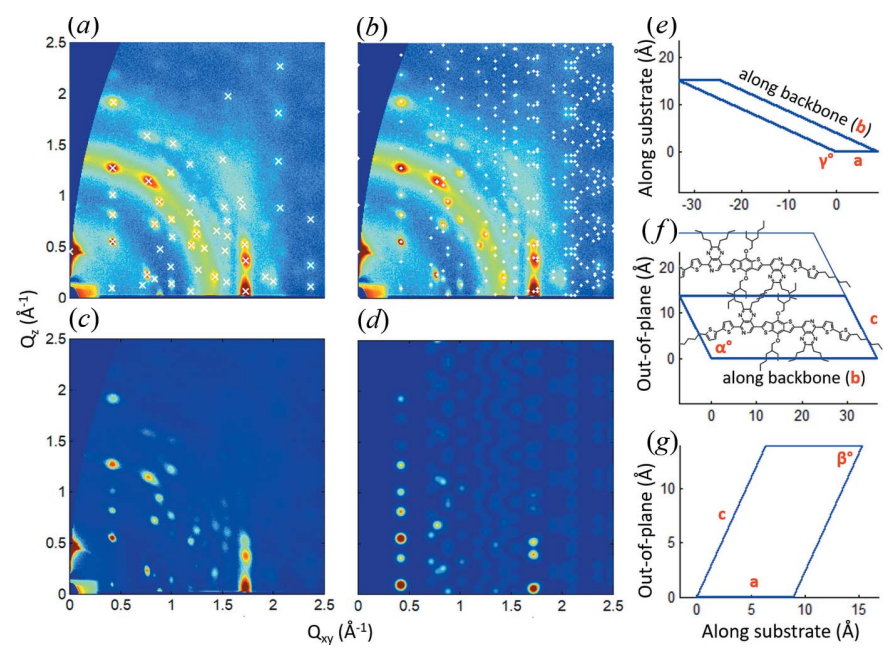

Figure 12

$(a),(b)$ GIWAXS pattern of SM1:PC ${ }_{71} \mathrm{BM}$, cast from chlorobenzene with $0.8 \%$ DIO in a $1: 1$ blend. (a) Crosses signify peak positions that were used for indexing. (b) Dots indicate the fitted scattering pattern. (c) Same data as (a), rescaled and with PCBM artificially removed. (d) Scattering pattern simulated using simDiffraction from a possible configuration of SM1 with the calculated unit cell. SM1 crystal structure: $(e)$ bird's-eye view of film; $(f),(g)$ side views from two different directions, perpendicular to the $b-c$ plane $(f)$ and $a-c$ plane $(g)$. In $(f)$, the molecular structure is drawn approximately to scale. to $(h k)=(10)$. For peak 2, we select the intense peak at $\left(Q_{x y}, Q_{z}\right)=(0.418,0.550) \AA^{-1}$. Our initial hypothesis is that peak 2, which has the lowest nonzero $Q_{x y}$ position observed, is the along-backbone peak, so we restrict it to an index of $(h k)=$ (01). All peaks are restricted to $h$ and $k$ values $\leq 8$. Thus, we initially attempt to solve the structure with a minimally sized structure by restricting peaks to low indices. However, we are prepared to loosen the restrictions if the accuracy of the generated scattering pattern is not satisfactory.

Any of the bright peaks in our scattering pattern are good candidates for peak 3 as long as their $Q_{x y}$ position is not a near multiple of the $Q_{x y}$ position of peak 2. After testing several of these, we determine that the peak at $\left(Q_{x y}, Q_{z}\right)=(0.761$, $0.229) \AA^{-1}$ produces the most accurate scattering pattern. These conditions give a reasonable peak position uncertainty of $\Delta Q_{x y}<0.04 \AA^{-1}$ for all peaks. The results are $a_{\|}^{*}=$ $1.716 \AA^{-1}, b_{\|}^{*}=0.418 \AA^{-1}$ and $\gamma_{\|}^{*}=24.32^{\circ}$.

For Step 2, we use a preliminary $c^{*}$ value of $0.46 \AA^{-1}$ based on the observed out-of-plane peak position (which we hypothesize is the alkyl peak). We select the bright peaks at $\left(Q_{x y}, Q_{z}\right)=(1.724,0.066) \AA^{-1}$ and $(0.416,0.093) \AA^{-1}$ for the initial calculation. We restrict the $\ell$ values of the first two peaks to 0 , forcing them to correspond to the primarily crystallographic directions (100) and (010) - or the $\pi-\pi$ stacking direction and the backbone direction. We choose the peak at low $Q_{z}$ as the along-backbone (010) peak because a structure with a backbone lying along the substrate is much more common in organic thin films than one where the backbone is angled upward (Osaka \& Takimiya, 2015).

With these restrictions in place, we obtain a reasonable peak position uncertainty of $\Delta Q<0.06 \AA^{-1}$ for all peaks (with smaller uncertainty for peaks at lower $Q$ values: e.g. for $Q_{x y}<$ $1.00 \AA^{-1}, \Delta Q<0.03 \AA^{-1}$ ), using $a_{\perp}^{*}=0.060 \AA^{-1}, b_{\perp}^{*}=$ $0.097 \AA^{-1}$ and $c^{*}=0.457 \AA^{-1}$. Our final crystal structure has
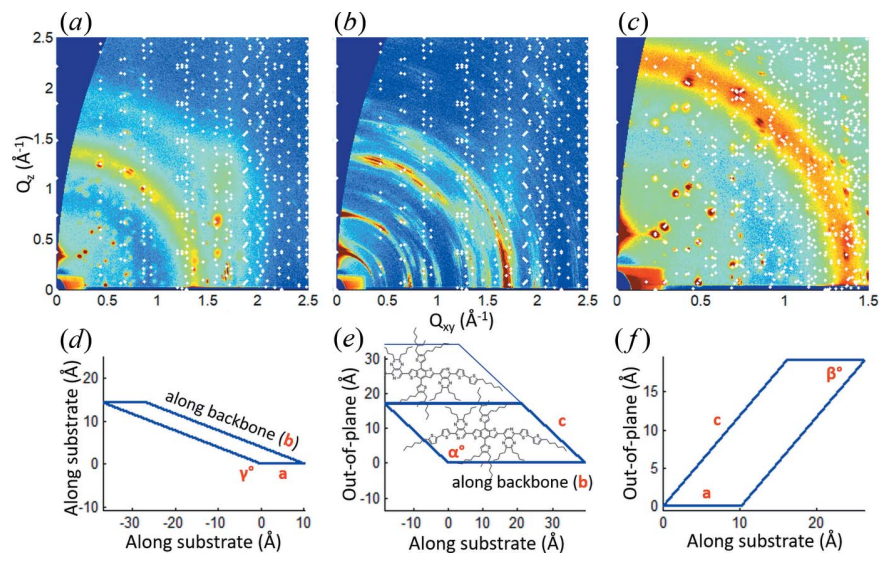

Figure 13

(a) GIWAXS pattern of SM2: $\mathrm{PC}_{71} \mathrm{BM}$, cast from chlorobenzene with $0.8 \%$ DIO in a $1: 1$ blend. Dots indicate the fitted scattering pattern for the first (lamellar) polymorph. (b) GIWAXS pattern of neat SM2, same conditions as $(a)$. (c) Same as $(a)$, zoomed-in and with a different color scale for detail, overlaid with the scattering pattern for the second polymorph which is not present in the neat film $(b)$. SM2 crystal structure: $(d)$ bird's-eye view of film; $(e),(f)$ side views from two different directions, perpendicular to the $b-c$ plane $(e)$ and $a-c$ plane $(f)$. In $(e)$, the molecular structure is drawn approximately to scale. 
$a=8.89, b=36.5, c=15.3 \AA, \alpha=115.9, \beta=67.4, \gamma=155.7^{\circ}$, and a volume of $1840 \AA^{3}$. The scattering pattern expected from this unit cell is shown in Fig. 12(b) and a visual representation of the crystal structure is shown in Figs. 12(e), 12(f) and 12(g).

To confirm this unit cell, we created crystallographic data files and used SimDiffraction (Breiby et al., 2008) to generate scattering patterns with intensities to compare with our experimental data. We tried several different configurations for the small molecule, which are shown in Fig. 20 (see Appendix $C$ ). All of these neglect hydrogen atoms (which have only a small effect on scattering intensity due to their low electron density) and side chains (which can rotate freely). The first three configurations have various thiophene orientations with a planar backbone while the fourth has a twisted backbone generated using the $Q$-chem ab initio quantum chemistry package (Shao et al., 2015). We used rotation matrices to orient the molecules as shown in Figs. 12(e), 12( $f)$ and $12(\mathrm{~g})$ and added the unit-cell parameters. The generated scattering patterns are shown in Fig. 20. Even the small changes to molecular structure that we tested have a substantial effect on the relative peak intensities. In general, however, all three configurations with a planar backbone replicate the high intensities of the $(10 \ell)$ peaks at $Q_{x y}=$ $1.7 \AA^{-1}$. The relative intensities of the $(10 \ell)$ peaks at $Q_{x y}=$ $0.4 \AA^{-1}$ are fairly accurate for the 'C-shaped' configuration shown in Fig. 20(b). A side-by-side comparison of our data (with fullerene scattering artificially subtracted) and the simulated pattern is shown in Figs. 12(c) and 12(d). The main discrepancy between the simulated pattern and what is experimentally observed is the intensity of the (010) peak at $Q_{z}=0.1 \AA^{-1}$. The lower experimental intensity of this peak may indicate some non-planarity in the backbone. Because the generated scattering pattern is close to our experimental result, and the unit cell has reasonable dimensions given what we know about our small molecule, no further iterations of the calculation are necessary.

The unit cell reveals information about the packing and orientation of the molecule that is not obvious from the scattering pattern. Some examples of distances that are of interest are shown in Fig. 14. Here, the unit-cell vectors are defined using standard convention: $\mathbf{v}_{1}, \mathbf{v}_{2}, \mathbf{v}_{3}$ have lengths $a, b$, $c$, respectively, and the reciprocal-lattice vectors $\mathbf{u}_{1}, \mathbf{u}_{2}, \mathbf{u}_{3}$ are normal to the $b-c, a-c$ and $a-b$ planes, respectively.

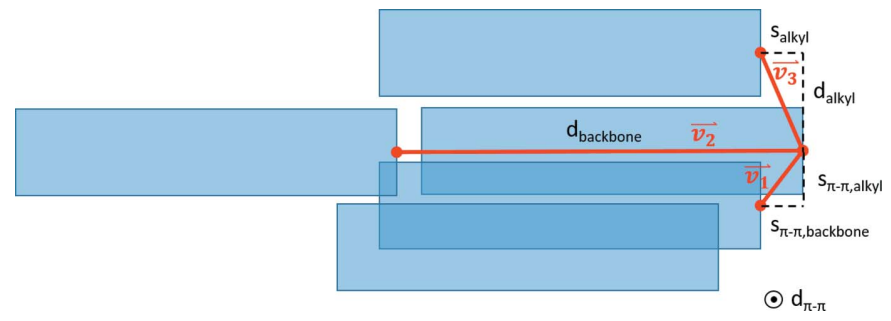

Figure 14

Schematic of distances that may be of interest for small molecules, oriented to view the backbone plane [similar to Fig. 12(f)]. Each rectangle represents a single planar small-molecule backbone; red lines show the unit-cell vectors.
The backbone length $d_{\text {backbone }}$ is simply $b=36.5 \AA$. The closest $\pi-\pi$ distance $d_{\pi-\pi}$ is the projection of $\mathbf{v}_{1}$ onto the vector normal to the backbone plane (the $b-c$ plane), which is the reciprocal-lattice vector $\mathbf{u}_{1}$. Thus,

$$
d_{\pi-\pi}=\frac{\mathbf{v}_{1} \cdot \mathbf{u}_{1}}{\left|\mathbf{u}_{1}\right|} .
$$

This equation gives $d_{\pi-\pi}=3.66 \AA$ for this unit cell.

It is also useful to calculate the slip-stacking of the $\pi-\pi$ stack, given as $s_{\pi-\pi, \text { backbone }}$ and $s_{\pi-\pi, \text { alkyl }}$ (see Fig. 14). The first step is to project $\mathbf{v}_{1}$ onto the backbone plane, using

$$
\mathbf{v}_{1, b-c}=\mathbf{v}_{1}-\frac{\mathbf{v}_{1} \cdot \mathbf{u}_{1}}{\left|\mathbf{u}_{1}\right|} \frac{\mathbf{u}_{1}}{\left|\mathbf{u}_{1}\right|} \text {. }
$$

Then, the components along and normal to the backbone direction $\mathbf{v}_{2}$ become

$$
\begin{aligned}
& s_{\pi-\pi, \text { backbone }}=\frac{\mathbf{v}_{1, b-c} \cdot \mathbf{v}_{2}}{\left|\mathbf{v}_{2}\right|} \text { and } \\
& s_{\pi-\pi, \text { alkyl }}=\left(\left|\mathbf{v}_{1, b-c}\right|^{2}-s_{\pi-\pi, \text { backbone }}^{2}\right)^{1 / 2} .
\end{aligned}
$$

These equations give $s_{\pi-\pi \text {,backbone }}=8.10 \AA$ and $s_{\pi-\pi, \text { alkyl }}=$ $0.13 \AA$.

We can similarly analyze the alkyl stacking distance and slip-stacking. Since $\mathbf{v}_{3}$ is already in the backbone plane (by definition),

$$
s_{\text {alkyl }}=\frac{\mathbf{v}_{3} \cdot \mathbf{v}_{2}}{\left|\mathbf{v}_{2}\right|} \text { and } d_{\text {alkyl }}=\left(\left|\mathbf{v}_{3}\right|^{2}-s_{\text {alkyl }}^{2}\right)^{1 / 2},
$$

or equivalently [using Fig. 12(f)],

$$
s_{\text {alkyl }}=c \cos \left(180^{\circ}-\alpha\right) \text { and } d_{\text {alkyl }}=c \sin \left(180^{\circ}-\alpha\right) \text {. }
$$

For this unit cell, the results are $s_{\text {alkyl }}=6.7 \AA$ and $d_{\text {alkyl }}=$ $13.8 \AA$.

Finally, the tilt of the backbone with respect to the substrate is of interest. The angle between the backbone plane and the substrate plane (the $a-b$ plane) is equal to the angle between $\mathbf{u}_{1}$ and $\mathbf{u}_{3}$. Thus,

$$
\text { Tilt angle }=\operatorname{acos} \frac{\mathbf{u}_{1} \cdot \mathbf{u}_{3}}{\left|\mathbf{u}_{1}\right|\left|\mathbf{u}_{3}\right|} .
$$

For this unit cell, this angle is found to be $88^{\circ}$ (with $90^{\circ}$ being edge-on).

Next, we examine SM2 (Fig. 13). The scattering pattern of SM2 has two unique peaks in the out-of-plane direction at $Q_{z}=$ 0.33 and $0.37 \AA^{-1}$. These are too far apart to be explained by refraction and they are unlikely to be multiples of a common $c^{*}$. This indicates two or more polymorphs present in SM2 that have to be indexed with different $c^{*}$ values. It is possible to index the two polymorphs separately because of the large number of peaks present. First, we extracted columns of peaks (same $Q_{x y}$ ) in the scattering pattern for which $\Delta Q_{z}$ spacings were equal to multiples of $0.37 \AA^{-1}$. This set of peaks could be indexed in a similar fashion to those of SM1 [see Figs. 13(a) and $13(b)]$. We assigned peaks at $\left(Q_{x y}, Q_{z}\right)=(1.70,0.20) \AA^{-1}$ and $(0.43,0.24) \AA^{-1}$ to the (001) and (010) indices, respectively. We also used the peak at $(1.36,0.21) \AA^{-1}$ for Step 1 , and restricted the $h, k$ values to $\leq 6$. The results of this indexing are given and compared with those of SM1 in Table 1. The 
Table 1

Crystal structure parameters of SM1 and SM2, from indexing of GIWAXS patterns.

See Fig. 14 for definitions of distances. For SM2, the lamellar polymorph corresponding to $c^{*}=0.37 \AA^{-1}$ is used.

\begin{tabular}{lllllllll}
\hline & & \multicolumn{3}{c}{ Tilt } & & & Unit-cell \\
& $d_{\text {backbone }}$ & $d_{\text {alkyl }}$ & $d_{\pi-\pi}$ & angle & $s_{\text {alkyl }}$ & $s_{\pi-\pi \text {,alkyl }}$ & $s_{\pi-\pi \text {,backbone }}$ & volume \\
\hline SM1 & $36.5 \AA$ & $13.8 \AA$ & $3.66 \AA$ & $88.0^{\circ}$ & $6.7 \AA$ & $0.1 \AA$ & $8.10 \AA$ & $1840 \AA^{3}$ \\
SM2 & $39.5 \AA$ & $17.1 \AA$ & $3.75 \AA$ & $82.1^{\circ}$ & $18.3 \AA$ & $0.5 \AA$ & $9.36 \AA$ & $2520 \AA^{3}$ \\
\hline
\end{tabular}

molecular length and $\pi-\pi$ stacking distances are similar between the two molecules, though SM2 has slightly larger parameters. The alkyl stacking distance is $3.3 \AA$ larger in SM2 which is consistent with the addition of two thiophenes in the side chains; this leads to a larger volume. SM2 is more slipstacked than SM1, especially along $s_{\text {alkyl }}$, which allows more efficient packing of the bulkier side chains. SM2 also has a smaller tilt angle, making it less edge on.

We then attempted to index the remainder of peaks in the SM2 pattern, corresponding to a $0.33 \AA^{-1}$ out-of-plane repeat distance $\left(c^{*}\right)$. The high density of peaks at low $Q$ indicates a large unit cell, probably containing several molecules [see Fig. 13(c)]. Indeed, the scattering pattern was most closely replicated by a unit cell with a volume of $18400 \AA^{3}, 7.5 \times$ larger than the unit cell of the other polymorph. The unit cell had parameters of $a=35.4, b=44.0, c=19.6 \AA, \alpha=78, \beta=105$, $\gamma=141^{\circ}$. Interestingly, the peaks and $c^{*}$ value associated with this polymorph were not present in the scattering patterns for the neat SM2 films, with or without DIO [see Fig. 13(b)]. This indicates that this large, well crystallized structure may contain both small molecules and fullerenes, such as in an intercalated structure (Miller et al., 2012). Alternatively, the larger structure may be due to a complex packing motif such as herringbone (Sherman et al., 2015) or slipped $\gamma$ (Yang et al., 2018) which is catalyzed by the presence of fullerene. Lastly, the peaks associated with the larger structure may actually arise from two or more additional polymorphs with the same out-of-plane repeat distance of $0.33 \AA^{-1}$. Solving for the exact structure in this case is difficult without further analysis using simulation or single-crystal diffraction.

\section{Related literature}

The following references are cited in the supporting information: Mateker et al. (2015), Cadek et al. (2010), Lide (2005), Mukherjee et al. (2017), Waldemar Knittel Glasbearbeitungs (2019), Medvedovski et al. (2008).

\section{Conclusions}

In this article, we have presented a software package called GIWAXS-SIIRkit geared towards helping researchers understand and analyze GIWAXS data of organic thin films. The name 'SIIRkit' reflects the fact that the package includes tools for examining relative scattering intensities (SI), indexing crystal patterns (I) and measuring refractive shifts to peak positions (R).

The scattering intensity in a GIWAXS measurement depends inter alia on the refractive index, which can be calculated using our separate refractive index tool; the electrical field strength within the film at a given incident angle; and the beam footprint on the sample. We present a toolbox to assess the changes to intensity when modifying sample composition and scattering geometry. This allows estimation of measurement uncertainty for a single sample and calculation of normalization factors for several samples of different sizes and/or compositions.

Refraction of the incident beam at grazing incidence will systematically shift observed peak positions to higher $Q$ (lower observed $d$ spacings). The refractive shift in the out-ofplane direction has been reported previously, and here we have expanded this calculation to include off-axis peak shifts. Calculating the refractive shift at various polar angles requires a straightforward but nontrivial procedure. For this reason we implement a simulation which can either calculate the actual $d$ spacing and repeat direction within a film given observed $\left(Q_{x y}\right.$, $\left.Q_{z}\right)$, or predict the observed $\left(Q_{x y}, Q_{z}\right)$ for a set of planes with user-defined $d$ spacing and repeat direction. Our simulation also predicts the location of an additional scattering peak due to reflection off the substrate.

The indexing tools that we have developed can be used to calculate the crystal structure of well ordered materials. The crystalline structure determines the intermolecular packing distances, which affect charge carrier mobility. In blended films, the crystal structure may also affect the interfacial interactions between a small molecule and another component such as a fullerene derivative. While some organic materials are crystalline enough to give rise to many peaks in a GIWAXS pattern, they are often too disordered to apply a traditional X-ray diffraction method.

In summary, we have developed a user-friendly software toolbox to help researchers understand their GIWAXS data and accurately report findings. This should enable better characterization of thin-film materials, which will improve the materials engineering process.

\section{APPENDIX $A$}

Calculation of the electric field within a thin film at grazing incidence

For convenience, we recreate the calculation of electric field within a thin film that was demonstrated by Factor (1991).

\section{A1. Setup variables}

We start with an incoming electric field that has components along $y$ (vertical) and $z$, using the convention of Fig. 8. We define the incoming wavevector as $k_{0}=\left(0 k_{y, 0} k_{z, 0}\right)$, the wavevector just inside the film as $k_{1}=\left(0 k_{y, 1} k_{z, 1}\right)$ and wavevectors in subsequent layers as $k_{i}=\left(0 k_{y, i} k_{z, i}\right)$. The magnitude of each wavevector is 


$$
\left|\mathbf{k}_{i}\right|=k_{i}=\left(k_{y, i}^{2}+k_{z, i}^{2}\right)^{1 / 2}=\omega n_{i} / c=k n_{i}
$$

with $\omega=$ angular frequency, $n_{i}=$ refractive index of the layer $=$ $1-\delta_{i}+i \beta_{i}$ ( $i$ being the imaginary unit), $c=$ speed of light and $k \equiv \omega / c$. Given that the medium outside the film is typically vacuum or an inert gas, with $n_{0}=1$, we can reasonably assume that $k_{0} \simeq k$.

\section{A2. Proof that $k_{y, i}$ is invariant}

The angle of the wavevector in each layer, with respect to the $X-Z$ plane (i.e. substrate surface), can be observed to follow $\sin \theta_{i}=k_{z, i} / k_{i}$. Snell's law states that

$$
n_{i} \cos \theta_{i}=n_{j} \cos \theta_{j}
$$

for any $i, j$ (note that we define $\theta$ relative to the surface plane; Snell's law is often given with $\theta$ relative to the normal of the surface plane).

A straightforward calculation yields $k_{y, i}=k_{y, j}$ for any $i, j$ :

$$
\begin{aligned}
k_{y, i} & =\left(k_{i}^{2}-k_{z, i}^{2}\right)^{1 / 2}=k_{i}\left[1-\left(\frac{k_{z, i}}{k_{i}}\right)^{2}\right]^{1 / 2} \\
& =k_{i}\left(1-\sin ^{2} \theta_{i}\right)^{1 / 2}=k_{i} \cos \theta_{i}=k_{j} \cos \theta_{j}=k_{y, j}
\end{aligned}
$$

For example, $k_{y, 0}=k_{y, 1}$, which we will use in the next section.

\section{A3. Calculation of $k_{z, i}$}

We can calculate the $z$ component of the wavevector inside the film with respect to the $z$ component of the wavevector incident on the film using equations (17) and (19), assuming that $n_{0}=1$ :

$$
\begin{aligned}
k_{z, 1}^{2} & =k_{1}^{2}-k_{y, 1}^{2}=\left(\omega n_{1} / c\right)^{2}-k_{y, 0}^{2} \\
& =\left(\omega n_{1} / c\right)^{2}-\left(k^{2}-k_{z, 0}^{2}\right)=\left(\omega n_{1} / c\right)^{2}-(\omega / c)^{2}+k_{z, 0}^{2} \\
& =k_{z, 0}^{2}+k^{2}\left(n_{1}^{2}-1\right) .
\end{aligned}
$$

Expanding, we get

$$
\begin{aligned}
n_{1}^{2} & =\left(1-\delta_{1}+i \beta_{1}\right)^{2} \\
& =\left(1-\delta_{1}+i \beta_{1}\right)-\delta_{1}\left(1-\delta_{1}+i \beta_{1}\right)+i \beta_{1}\left(1-\delta_{1}+i \beta_{1}\right) \\
& =1-2 \delta_{1}+2 i \beta_{1}+\left(\delta_{1}^{2}-\beta_{1}^{2}-2 i \delta_{1} \beta_{1}\right) .
\end{aligned}
$$

Both $\delta$ and $\beta$ are typically small terms. As demonstrated in Table S1, $\delta$ is on the order of $10^{-6}$ and $\beta$ is on the order of $10^{-9}$. Thus, we can ignore the second-order terms. Furthermore, we observe that the critical angle in layer $i$ is $\alpha_{c, i}=\left(2 \delta_{i}\right)^{1 / 2}$, so that the critical wavevector is $k_{c, i}=k\left(2 \delta_{i}\right)^{1 / 2}$. With these observations, equation (20) turns into

$$
k_{z, 1}^{2}=k_{z, 0}^{2}+k^{2}\left(-2 \delta_{1}+2 i \beta_{1}\right)=k_{z, 0}^{2}-k_{c, 1}^{2}+2 i \beta_{1} k^{2} .
$$

If we let $k_{z, 1}=k_{z, 1, \operatorname{Re}}+i k_{z, 1, \mathrm{Im}}$, we can solve for the real and imaginary components:

$$
\begin{aligned}
k_{z, 1}^{2}= & \left(k_{z, 0}^{2}-k_{c, 1}^{2}\right)+2 i\left(\beta_{1} k^{2}\right) \\
= & \left(k_{z, 1, \operatorname{Re}}^{2}-k_{z, 1, \operatorname{Im}}^{2}\right)+2 i\left(k_{z, 1, \operatorname{Re}} k_{z, 1, \operatorname{Im}}\right) ; \\
k_{z, 1, \operatorname{Re}}^{2}= & \frac{k_{z, 0}^{2}-k_{c, 1}^{2} \pm\left[\left(k_{c, 1}^{2}-k_{z, 0}^{2}\right)^{2}+4 \beta_{1}^{2} k^{4}\right]^{1 / 2}}{2} ; \\
k_{z, 1, \operatorname{Im}}^{2}= & \frac{-k_{z, 0}^{2}+k_{c, 1}^{2} \pm\left[\left(k_{c, 1}^{2}-k_{z, 0}^{2}\right)^{2}+4 \beta_{1}^{2} k^{4}\right]^{1 / 2}}{2} .
\end{aligned}
$$

The top sign (+) corresponds to an attenuating field while the bottom sign (-) gives an amplifying field. This means only the top sign gives a result consistent with energy conservation.

A similar derivation can be used to compare the wavefunction of any layer with that of the incident beam $k_{0}$. The equivalent of equation (22) will be

$$
k_{z, i}^{2}=k_{z, 0}^{2}-k_{c, i}^{2}+2 i \beta_{i} k^{2},
$$

which yields an equation with the same format as equation (23), with the subscript 1 replaced by $i$. Thus we arrive at equation (5) in the main text, where we have removed the subscript $z$ for legibility.

\section{A4. Calculating the reflectance at an interface}

If $z=0$ is the top surface of the film, the electric field outside the film $(z<0)$ is a sum of the incident and reflected waves:

$$
E=E_{\mathrm{i}} \exp \left[i\left(k_{y, 0} y+k_{z, 0} z\right)\right]+E_{\mathrm{r}} \exp \left[i\left(k_{y, 0} y-k_{z, 0} z\right)\right],
$$

where $E_{\mathrm{i}}$ and $E_{\mathrm{r}}$ are the field strengths of the incident and reflected beams, respectively. The electric field propagating into the film $(z>0)$ is given as

$$
E=E_{\mathrm{t}} \exp \left[i\left(k_{y, 1} y+k_{z, 1} z\right)\right]
$$

where $E_{\mathrm{t}}$ is the field strength of the transmitted beam. The continuity condition of $E(y, z)$ and $[\partial E(y, z)] / \partial z$ must be met at the interface $(z=0)$, i.e.

$$
\begin{aligned}
& E_{\mathrm{i}} \exp \left[i\left(k_{y, 0} y+k_{z, 0} z\right)\right]+E_{\mathrm{r}} \exp \left[i\left(k_{y, 0} y-k_{z, 0} z\right)\right] \\
& \quad=E_{\mathrm{t}} \exp \left[i\left(k_{y, 1} y+k_{z, 1} z\right)\right] \quad \text { at } \quad z=0 \quad \text { and } \\
& \frac{\partial}{\partial z}\left\{E_{\mathrm{i}} \exp \left[i\left(k_{y, 0} y+k_{z, 0} z\right)\right]+E_{\mathrm{r}} \exp \left[i\left(k_{y, 0} y-k_{z, 0} z\right)\right]\right\} \\
& =\frac{\partial}{\partial z}\left\{E_{\mathrm{t}} \exp \left[i\left(k_{y, 1} y+k_{z, 1} z\right)\right]\right\} \quad \text { at } z=0 .
\end{aligned}
$$

Solving and rearranging (keeping in mind that $k_{y, 0}=k_{y, 1}$ ),

$$
E_{\mathrm{i}}+E_{\mathrm{r}}=E_{\mathrm{t}} \quad \text { and } \quad\left(E_{\mathrm{i}}-E_{\mathrm{r}}\right) k_{z, 0}=E_{\mathrm{t}} k_{z, 1} .
$$

Equation (28) can be used to solve for the Fresnel reflection $r_{01}\left(\right.$ defined as $E_{\mathrm{r}} / E_{\mathrm{i}}$ ) and transmission coefficient $t_{01}$ (defined as $\left.E_{\mathrm{t}} / E_{i}\right)$ at the interface:

$$
\begin{aligned}
& r_{01}=\frac{E_{\mathrm{r}}}{E_{\mathrm{i}}}=\frac{k_{z, 0}-k_{z, 1}}{k_{z, 0}+k_{z, 1}} \text { and } \\
& t_{01}=\frac{E_{\mathrm{t}}}{E_{\mathrm{i}}}=\frac{2 k_{z, 0}}{k_{z, 0}+k_{z, 1}}=r_{01}+1 .
\end{aligned}
$$

This solution can be generalized for any interface in a series of stacked parallel layers, yielding the solution for $r_{12}$ given in equation (4) of the main text. 


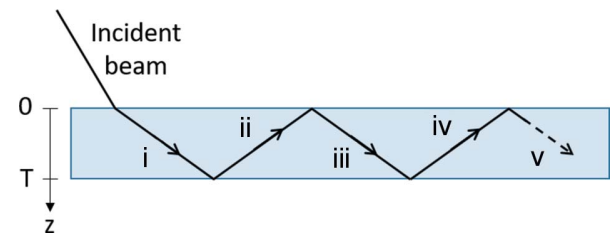

Figure 15

Schematic of beam reflection within a thin film. Angles are exaggerated for clarity.

\section{A5. Calculating the electric field in the film}

We derive the equation for the $z$ component of the electric field using similar methods to those of Borne \& Wolfe (1985). After entering the thin film, the electric field fluctuates back and forth as shown in Fig. 15, losing energy at each interface. The total electric field can be written as the sum of the components indicated in Fig. 15:

$$
E=\mathrm{i}+\mathrm{ii}+\mathrm{iii}+\mathrm{iv}+\mathrm{v}+\cdots .
$$

The electric field that is transmitted into the film can be written as in the previous section:

$$
\begin{aligned}
\mathrm{i} & =E_{\mathrm{t}} \exp \left[i\left(k_{z, 1} z\right)\right]=E_{\mathrm{i}} t_{01} \exp \left[i\left(k_{z, 1} z\right)\right] \\
& =E_{\mathrm{i}}\left(1+r_{01}\right) \exp \left[i\left(k_{z, 1} z\right)\right] .
\end{aligned}
$$

At the bottom interface, the field will be reflected with a coefficient $r_{12}$. Reflection will require replacing $z$ with $(2 T-z)$ so that $E(T) \propto \exp \left[i\left(k_{z, 1} T\right)\right]$ and $E(0) \propto$ $\exp \left[i\left(k_{z, 1} 2 T\right)\right]$ (= two traversals through the film). This gives

$$
\begin{aligned}
\mathrm{ii} & =E_{\mathrm{i}}\left(1+r_{01}\right) r_{12} \exp \left\{i\left[k_{z, 1}(2 T-z)\right]\right\} \\
& =E_{\mathrm{i}}\left(1+r_{01}\right)\left\{r_{12} \exp \left[i\left(k_{z, 1} 2 T\right)\right]\right\} \exp \left[-i\left(k_{z, 1} z\right)\right] .
\end{aligned}
$$

At the top surface, reflection will add a coefficient $r_{10}=-r_{01}$ and require replacing $(2 T-z)$ with $(z+2 T)$ so that $E(0) \propto \exp \left[i\left(k_{z, 1} 2 T\right)\right]$ (two traversals) and $E(T) \propto$ $\exp \left[i\left(k_{z, 1} 3 T\right)\right]$ (= three traversals):

$$
\begin{aligned}
\text { iii } & =E_{\mathrm{i}}\left(1+r_{01}\right) r_{12}\left(-r_{01}\right) \exp \left\{i\left[k_{z, 1}(z+2 T)\right]\right\} \\
& =E_{\mathrm{i}}\left(1+r_{01}\right)\left\{-r_{01} r_{12} \exp \left[i\left(k_{z, 1} 2 T\right)\right]\right\} \exp \left[i\left(k_{z, 1} z\right)\right] .
\end{aligned}
$$

We can repeat this procedure to find

$$
\begin{gathered}
\mathrm{iv}=E_{\mathrm{i}}\left(1+r_{01}\right)\left\{-r_{01} r_{12}^{2} \exp \left[i\left(k_{z, 1} 4 T\right)\right]\right\} \exp \left[-i\left(k_{z, 1} z\right)\right] \\
\mathrm{v}=E_{\mathrm{i}}\left(1+r_{01}\right)\left\{r_{01}^{2} r_{12}^{2} \exp \left[i\left(k_{z, 1} 4 T\right)\right]\right\} \exp \left[i\left(k_{z, 1} z\right)\right]
\end{gathered}
$$

and so on. Summing all these components together, we find

$$
\begin{aligned}
E= & E_{\mathrm{i}}\left(1+r_{01}\right) \\
& \times\left\{\zeta \exp \left[i\left(k_{z, 1} z\right)\right]+\zeta r_{12} \exp \left[i\left(k_{z, 1} 2 T\right)\right] \exp \left[-i\left(k_{z, 1} z\right)\right]\right\},
\end{aligned}
$$

with

$$
\zeta=1-r_{01} r_{12} \exp \left[i\left(k_{z, 1} 2 T\right)\right]+r_{01}^{2} r_{12}^{2} \exp \left[i\left(k_{z, 1} 4 T\right)\right]-\cdots .
$$

We recognize the term $\zeta$ as the Taylor expansion form of $1 /(1+x)$ with $x=r_{01} r_{12} \exp \left[i\left(k_{z, 1} 2 T\right)\right]$. Thus we can write

$$
\begin{aligned}
& E=E_{\mathrm{i}}\left(1+r_{01}\right) \\
& \times \frac{\exp \left[i\left(k_{z, 1} z\right)\right]+r_{12} \exp \left[i\left(k_{z, 1} 2 T\right)\right] \exp \left[-i\left(k_{z, 1} z\right)\right]}{1+r_{01} r_{12} \exp \left[i\left(k_{z, 1} 2 T\right)\right]},
\end{aligned}
$$

which is equivalent to equation (4) in the main text. This result is identical to that obtained using the formalism in the work of Dev et al. (2000) with a single layer on a substrate and negligible surface roughness.

\section{A6. Demonstration of electric field in a film}

The dependence of electric field on incident angle has been demonstrated previously (Factor, 1991). We include some results here for convenience, along with the dependence on film thickness. This is meant to illustrate why the scattering intensity is non-monotonic with thickness. We use the refractive indices of a neat PTB7 film on a silicon substrate (see Table S1). The critical angles of PTB7 and silicon are 0.1005 and $0.1405^{\circ}$, respectively.

The electric field within a $100 \mathrm{~nm}$ film is shown in Fig. 16(a). For incident angles below the film's critical angle (0.08 and $\left.0.10^{\circ}\right)$, the beam intensity within the film follows an exponential fall-off. Above the film's critical angle but below the substrate's critical angle $\left(0.12\right.$ and $\left.0.14^{\circ}\right)$, a large fraction of the beam enters the film but is reflected off the substrate, resulting in a high-intensity standing wave within the film and boosting scattering intensity. Above both critical angles (0.16 and $0.30^{\circ}$ ), a standing wave exists in the film but some intensity passes into the substrate and is lost.

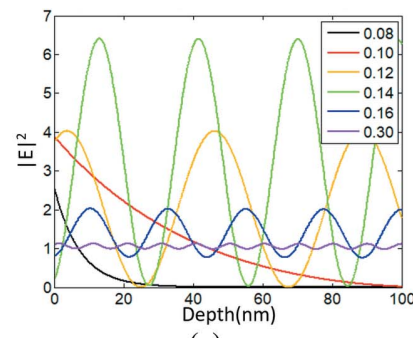

(a)

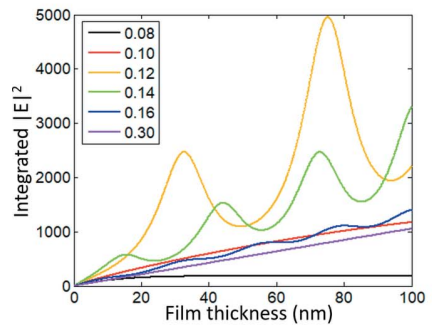

(c)

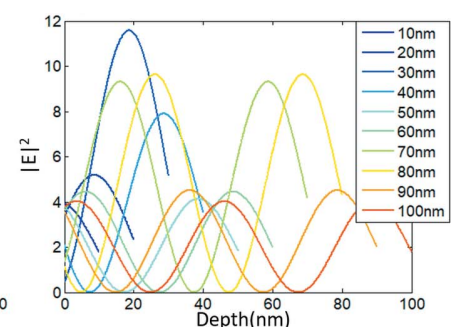

(b)

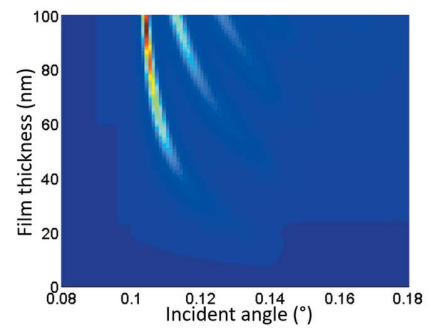

(d)
Figure 16

(a) Squared electric field $|E|^{2}$ within a $100 \mathrm{~nm}$ PTB7 thin film on top of a silicon substrate, with various incident angles, as labeled in the legend (in $\left.{ }^{\circ}\right)$. (b) $|E|^{2}$ for films of varying thicknesses, as shown in the legend, at an incident angle of $0.12^{\circ}$. (c) Integrated electric field as a function of film thickness for various incident angles. Each point corresponds to the integral of $|E|^{2}$, generated at the given incident angle and thickness, from a depth of 0 to $T$ : i.e. the area under the curves in $(b) .(d) \int_{0}^{T}|E|^{2} \mathrm{~d} z$ versus incident angle and thickness. 
Because the electric field is reflected within the film, $|E|^{2}$ depends on the film thickness. The electric field for films of varying thickness, with an incident angle of $0.12^{\circ}$, is shown in Fig. 16(b).

The integral of $|E|^{2}$ over the depth of the film is proportional to the scattering intensity. The dependence of the integrated electric field on the film thickness and incident angle is shown in Fig. 16(c). At low incident angles $\left(0.08^{\circ}\right)$, the electric field falls off within the top $5-10 \mathrm{~nm}$ and so the integrated electric field is independent of film thickness. At intermediate incident angles $\left(0.12,0.14^{\circ}\right)$, there is a standing wave with a period comparable to the film thickness, which causes the fringing in Fig. 16(c). At high incident angles $\left(0.30^{\circ}\right)$, this fringing is diminished because lower-intensity reflected beams interfere less with the incident beam, and the dependence of scattering intensity on thickness approached a linear relationship.

Thus, $\int_{0}^{T}|E|^{2} \mathrm{~d} z$ is highly nonlinear with both incident angle and film thickness, giving rise to the fringe pattern in Fig. 16(d). The intensity peaks near the film critical angle and eventually plateaus at higher angles.

We also compare the results of our calculation with those of the TER_sl program, which can generate a plot of electric field strength within a thin film (Stepanov, 2019, 2004). This program uses recursive matrix operations which are demonstrated in the work of Stepanov et al. (1998). The results of this program, given the same parameters as used in Fig. 16, are nearly identical to our results.

\section{APPENDIX $B$}

\section{Calculation of refractive shift in two dimensions}

\section{B1. Useful relationships}

From the definition of $Q$, it can be observed that

$$
Q=\frac{4 \pi}{\lambda} \sin \frac{2 \theta}{2} \text {. }
$$

The polar angle $\chi$ is defined such that

$$
\tan \chi=Q_{z} / Q_{x y}
$$

or $\sin \chi=Q_{z} / Q$. This is equivalent to $90^{\circ}$, the angle between $\mathbf{Q}$ and the surface normal:

$$
\sin \chi=\cos \left(90^{\circ}-\chi\right)=\frac{\mathbf{Q}}{|\mathbf{Q}|} \cdot\left(\begin{array}{l}
0 \\
0 \\
1
\end{array}\right)=\frac{Q_{z}}{Q},
$$

where indicates a dot product. In other words, the polar angle gives the tilt angle between the diffracting planes and the substrate surface, such that $\chi=0^{\circ}$ implies periodicity along the in-plane direction and $\chi=90^{\circ}$ means periodicity along the outof-plane direction.

Bragg's law states that

$$
d=\frac{\lambda}{2 \sin \left(2 \theta_{\mathrm{B}} / 2\right)} \leftrightarrow \sin \left(2 \theta_{\mathrm{B}} / 2\right)=\frac{\lambda}{2 d} .
$$

B2. An expression for $2 \theta$ in terms of vertical angles $\left(\theta_{1}, \theta_{1 \mathrm{~s}, Z}\right)$ and $\chi$

We start with an expression for the incident and scattered vectors similar to that given by Hexemer \& MüllerBuschbaum (2015):

$$
\mathbf{k}_{1}=\frac{2 \pi}{\lambda}\left(\begin{array}{c}
\cos \theta_{1} \\
0 \\
-\sin \theta_{1}
\end{array}\right) \text { and } \mathbf{k}_{1 \mathrm{~s}}=\frac{2 \pi}{\lambda}\left(\begin{array}{c}
\cos \theta_{1 \mathrm{~s}, X Y} \cos \theta_{1 \mathrm{~s}, Z} \\
\sin \theta_{1 \mathrm{~s}, X Y} \cos \theta_{1 \mathrm{~s}, Z} \\
\sin \theta_{1 \mathrm{~s}, Z}
\end{array}\right) \text {. }
$$

$\mathbf{Q}$ is defined such that

$\mathbf{Q}=\mathbf{k}_{1 \mathrm{~s}}-\mathbf{k}_{1}=\frac{2 \pi}{\lambda}\left(\begin{array}{c}\cos \theta_{1 \mathrm{~s}, X Y} \cos \theta_{1 \mathrm{~s}, Z}-\cos \theta_{1} \\ \sin \theta_{1 \mathrm{~s}, X Y} \cos \theta_{1 \mathrm{~s}, Z} \\ \sin \theta_{1 \mathrm{~s}, Z}+\sin \theta_{1}\end{array}\right)=\left(\begin{array}{c}Q_{x} \\ Q_{y} \\ Q_{z}\end{array}\right)$.

$Q_{x y}$ can be calculated as

$$
\begin{aligned}
Q_{x y}= & \left(Q_{x}^{2}+Q_{y}^{2}\right)^{1 / 2} \\
= & \frac{2 \pi}{\lambda}\left(\cos ^{2} \theta_{1 \mathrm{~s}, X Y} \cos ^{2} \theta_{1 \mathrm{~s}, Z}-2 \cos \theta_{1 \mathrm{~s}, X Y} \cos \theta_{1 \mathrm{~s}, Z} \cos \theta_{1}\right. \\
& \left.+\cos ^{2} \theta_{1}+\sin ^{2} \theta_{1 \mathrm{~s}, X Y} \cos ^{2} \theta_{1 \mathrm{~s}, Z}\right)^{1 / 2} \\
= & \frac{2 \pi}{\lambda}\left(\cos ^{2} \theta_{1 \mathrm{~s}, Z}+\cos ^{2} \theta_{1}-2 \cos \theta_{1 \mathrm{~s}, X Y} \cos \theta_{1 \mathrm{~s}, Z} \cos \theta_{1}\right)^{1 / 2} .
\end{aligned}
$$

The scattering angle can be defined using a vector dot product:

$$
\begin{aligned}
\cos \left(2 \theta_{1}\right) & =\frac{\mathbf{k}_{1} \cdot \mathbf{k}_{1 \mathrm{~s}}}{\left|\mathbf{k}_{1}\right|\left|\mathbf{k}_{1 \mathrm{~s}}\right|} \\
& =\cos \theta_{1} \cos \theta_{1 \mathrm{~s}, X Y} \cos \theta_{1 \mathrm{~s}, Z}-\sin \theta_{1} \sin \theta_{1 \mathrm{~s}, Z}
\end{aligned}
$$

We can combine equation (39) with equation (45) to eliminate the $\cos \theta_{1 \mathrm{~s}, X Y}$ term. By rearranging, we find that

$$
\begin{aligned}
& \tan \chi_{1}=Q_{z} / Q_{x y} \\
& =\frac{\sin \theta_{1 \mathrm{~s}, Z}+\sin \theta_{1}}{\left(\cos ^{2} \theta_{1 \mathrm{~s}, Z}+\cos ^{2} \theta_{1}-2 \cos \theta_{1 \mathrm{~s}, X Y} \cos \theta_{1 \mathrm{~s}, Z} \cos \theta_{1}\right)^{1 / 2}}, \\
& \cos ^{2} \theta_{1 \mathrm{~s}, Z}+\cos ^{2} \theta_{1}-2 \cos \theta_{1 \mathrm{~s}, X Y} \cos \theta_{1 \mathrm{~s}, Z} \cos \theta_{1} \\
& =\frac{\left(\sin \theta_{1 \mathrm{~s}, Z}+\sin \theta_{1}\right)^{2}}{\tan ^{2} \chi_{1}}, \\
& \frac{1}{2}\left[\begin{array}{c}
\left.\cos ^{2} \theta_{1 \mathrm{~s}, Z}+\cos ^{2} \theta_{1}-\frac{\left(\sin \theta_{1 \mathrm{~s}, Z}+\sin \theta_{1}\right)^{2}}{\tan ^{2} \chi_{1}}\right] \\
=\cos \theta_{1 \mathrm{~s}, X Y} \cos \theta_{1 \mathrm{~s}, Z} \cos \theta_{1} .
\end{array}\right.
\end{aligned}
$$

Substituting equation (46) into equation (45), we see that

$$
\begin{aligned}
\cos \left(2 \theta_{1}\right)= & \frac{1}{2}\left[\cos ^{2} \theta_{1 \mathrm{~s}, Z}+\cos ^{2} \theta_{1}-\frac{\left(\sin \theta_{1 \mathrm{~s}, Z}+\sin \theta_{1}\right)^{2}}{\tan ^{2} \chi_{1}}\right] \\
& -\sin \theta_{1} \sin \theta_{1 \mathrm{~s}, Z} .
\end{aligned}
$$


This expression relates the scattering angles inside the film or without refraction. We can similarly find an expression for variables outside the film:

$$
\begin{aligned}
\cos \left(2 \theta_{0}\right)= & \frac{1}{2}\left[\cos ^{2} \theta_{0 \mathrm{~s}, Z}+\cos ^{2} \theta_{0}-\frac{\left(\sin \theta_{0 \mathrm{~s}, Z}+\sin \theta_{0}\right)^{2}}{\tan ^{2} \chi_{0}}\right] \\
& -\sin \theta_{0} \sin \theta_{0 \mathrm{~s}, Z},
\end{aligned}
$$

where $2 \theta_{0}$ and $\chi_{0}$ can be directly converted to the observed $Q_{x y}, Q_{z}$ locations using equations (38) and (39).

Equations (47) and (48) can be rearranged into a quadratic form to solve for $\theta_{1 \mathrm{~s}, Z}$ or $\theta_{0 \mathrm{~s}, Z}$ :

$$
\begin{gathered}
\xi^{2}+\xi\left(2 \sin \theta_{1}\right)+\left[\left(2 \cos 2 \theta_{1}-1\right) \sin ^{2} \chi_{1}\right. \\
\left.+\sin \left(\theta_{1}-\chi_{1}\right) \sin \left(\theta_{1}+\chi_{1}\right)\right]=0
\end{gathered}
$$

(with $\xi=\sin \theta_{1 \mathrm{~s}, Z}$ ), and

$$
\begin{aligned}
& \varsigma^{2}+\varsigma\left(2 \sin \theta_{0}\right)+\left[\left(2 \cos 2 \theta_{0}-1\right) \sin ^{2} \chi_{0}\right. \\
& \left.+\sin \left(\theta_{0}-\chi_{0}\right) \sin \left(\theta_{0}+\chi_{0}\right)\right]=0
\end{aligned}
$$

(with $\left.\varsigma=\sin \theta_{0 \mathrm{~s}, Z}\right)$.

\section{B3. Applying refractive shift to vertical angles}

To apply refractive shift to the incoming beam $\left(\theta_{0} \rightarrow \theta_{1}\right)$ and the exit beam $\left(\theta_{1 \mathrm{~s}, Z} \rightarrow \theta_{0 \mathrm{~s}, Z}\right)$, we use the same approximations as Toney \& Brennan (1989):

$$
\begin{aligned}
& \theta_{1}=\frac{1}{2^{1 / 2}}\left\{\left[\left(\theta_{0}^{2}-2 \delta\right)^{2}+4 \beta^{2}\right]^{1 / 2}+\theta_{0}^{2}-2 \delta\right\}^{1 / 2} \leftrightarrow \\
& \theta_{0}=\left(\theta_{1}^{2}-\frac{\beta^{2}}{\theta_{1}^{2}}+2 \delta\right)^{1 / 2}
\end{aligned}
$$

and

$$
\begin{aligned}
& \theta_{1 \mathrm{~s}, Z}=\frac{1}{2^{1 / 2}}\left\{\left[\left(\theta_{0 \mathrm{~s}, Z}^{2}-2 \delta\right)^{2}+4 \beta^{2}\right]^{1 / 2}+\theta_{0 \mathrm{~s}, Z}^{2}-2 \delta\right\}^{1 / 2} \leftrightarrow \\
& \theta_{0 \mathrm{~s}, Z}=\left(\theta_{1 \mathrm{~s}, Z}^{2}-\frac{\beta^{2}}{\theta_{1 \mathrm{~s}, Z}^{2}}+2 \delta\right)^{1 / 2} .
\end{aligned}
$$

The refractive shift does not change the horizontal scattering angle: $\theta_{1 \mathrm{~s}, X Y}=\theta_{0 \mathrm{~s}, X Y}=\theta_{\mathrm{s}, X Y}$. The refractive shift will change the observed polar angle $\chi$ :

$$
\tan \chi_{1}=\frac{\sin \theta_{1 \mathrm{~s}, Z}+\sin \theta_{1}}{\left(\cos ^{2} \theta_{1 \mathrm{~s}, Z}+\cos ^{2} \theta_{1}-2 \cos \theta_{\mathrm{s}, X Y} \cos \theta_{1 \mathrm{~s}, Z} \cos \theta_{1}\right)^{1 / 2}}
$$

and

$$
\tan \chi_{0}=\frac{\sin \theta_{0 \mathrm{~s}, Z}+\sin \theta_{0}}{\left(\cos ^{2} \theta_{0 \mathrm{~s}, Z}+\cos ^{2} \theta_{0}-2 \cos \theta_{\mathrm{s}, X Y} \cos \theta_{0 \mathrm{~s}, Z} \cos \theta_{0}\right)^{1 / 2}}
$$

B4. Special situations: along-axis and sub-critical-angle scattering

In a real scattering situation, scattering along the $Q_{z}$ axis $\left(Q_{x y}=0\right.$ or $\left.\theta_{0 \mathrm{~s}, Z}=\theta_{0}\right)$ cannot be observed due to the curvature of the Ewald sphere versus the planar detector, giving rise to the inaccessible 'wedge' observed in the out-of-plane direction. However, a broad peak may be extrapolated to have a position at $Q_{x y}=0$. This does not cause a problem with the calculation procedure: no divide-by-zero issues arise.

Scattering along the $Q_{x y}$ axis is also unphysical because $Q_{z}=(2 \pi / \lambda)\left(\sin \theta_{0 \mathrm{~s}, Z}+\sin \theta_{0}\right)=0$ requires a negative scattering angle $\theta_{0 \mathrm{~s}, Z}$ (i.e. scattering in the $-Z$ direction). In fact, this occurs whenever $Q_{z}<(2 \pi / \lambda) \sin \theta_{0}$. In practice, there is often an increase in intensity observed along the Yoneda peak (Vineyard, 1982), but we do not consider this effect in this work. Another problem arises if either of the exterior angles is smaller than the critical angle of the film. If the incident angle is less than the critical angle, total internal reflection occurs (the real part of $\theta_{1}$ is $0^{\circ}$ ); this does not cause any computational problems. In this case there will not be a reflected peak. However, an exit angle that is smaller than the critical angle is non-physical; this will occur if a user enters $Q_{z}<(2 \pi / \lambda)\left(\sin \theta_{\text {critical }}+\sin \theta_{0}\right)$. To handle both these problems, we reset $Q_{z}$ to this limit $\left[Q_{z}=(2 \pi / \lambda) \times\right.$ $\left.\left(\sin \theta_{\text {critical }}+\sin \theta_{0}\right)\right]$ when necessary.

If $\chi_{1}$ is set to be close to 0 , there will be problems in applying equation (52) to calculate $\theta_{0 \mathrm{~s}, Z}$. In particular, if $\theta_{1 \mathrm{~s}, Z}^{2}-\left(\beta^{2} / \theta_{1 \mathrm{~s}, Z}^{2}\right)+2 \delta<0$ or $\theta_{1 \mathrm{~s}, Z}<\left[-\delta+\left(\delta^{2}+\beta^{2}\right)^{1 / 2}\right]^{1 / 2}$ very small number), then the calculated $\theta_{0 \mathrm{~s}, Z}$ will be imaginary. To avoid this problem, we take the absolute value of the result.

If $\chi_{1}$ is set to be exactly 0 , a divide-by-zero error will occur when applying equation (53) to calculate $\theta_{\mathrm{s}, X Y}$. Instead of using equation (53) in this special situation, we recalculate starting from the dot-product definition, using the relation $\sin \theta_{1 \mathrm{~s}, Z}+\sin \theta_{1}=0$ :

$$
\begin{aligned}
\cos \left(2 \theta_{1}\right) & =\cos \theta_{1} \cos \theta_{\mathrm{s}, X Y} \cos \theta_{1 \mathrm{~s}, Z}-\sin \theta_{1} \sin \theta_{1 \mathrm{~s}, Z} \\
& =\cos \theta_{1} \cos \theta_{\mathrm{s}, X Y} \cos \left(-\theta_{1}\right)-\sin \theta_{1} \sin \left(-\theta_{1}\right) \\
& =\cos ^{2} \theta_{1} \cos \theta_{\mathrm{s}, X Y}+\sin ^{2} \theta_{1} .
\end{aligned}
$$

We use this relation instead of equation (53).

\section{B5. Full procedure for calculation}

The equations presented here can be used to calculate the $d$ spacing and scattering angle $\chi_{1}$ from observed $\left(Q_{x y}, Q_{z}\right)$ positions and vice versa. For convenience, we have included flowcharts on how to apply these equations for both cases in Fig. 17 and Fig. 18. Green shading denotes input variables (supplied by the user) and orange denotes output variables.

B6. Comparison of the results of our simulation with those of Resel et al. (2016)

We modified the procedure somewhat in order to compare the results of our equations with the scattering pattern observed by Resel et al. (2016). This work assumed a direct conversion between the out-of-plane scattering angle and $Q_{z}$. In fact, $Q_{z}$ is defined in terms of $Q(2 \theta)$ and the polar angle $\chi$, making its relationship to the out-of-plane scattering angle less straightforward as shown above; however, this does not dramatically change the result. 


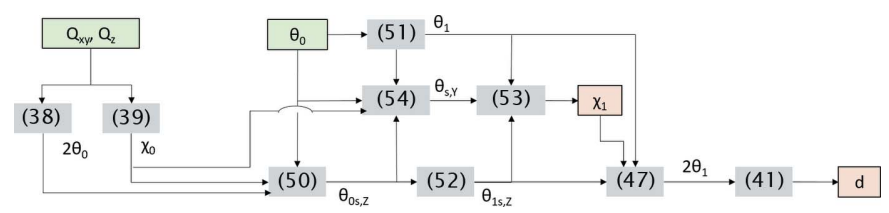

Figure 17

Flowchart showing $\left(Q_{x y}, Q_{z}, \theta_{0}, \lambda, \beta, \delta\right) \rightarrow\left(d\right.$ spacing, $\left.\chi_{1}\right)$ conversion. Equation numbers are given in parentheses.

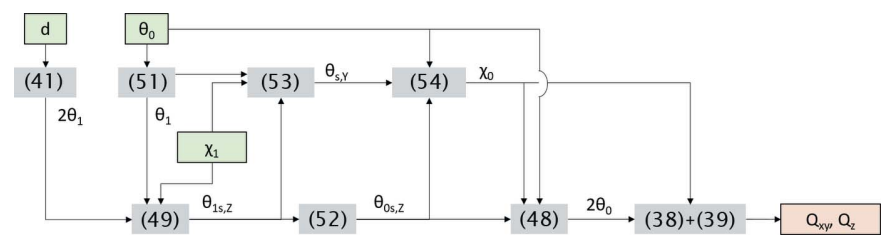

Figure 18

Flowchart showing ( $d$ spacing, $\left.\chi_{1}, \theta_{0}, \lambda, \beta, \delta\right) \rightarrow\left(Q_{x y}, Q_{z}\right)$ conversion. If $\chi_{1}=0$, equation (53) will be replaced with equation (55), which has inputs of $2 \theta_{1}$ and $\theta_{1}$ and outputs $\theta_{\mathrm{s}, X Y}$.

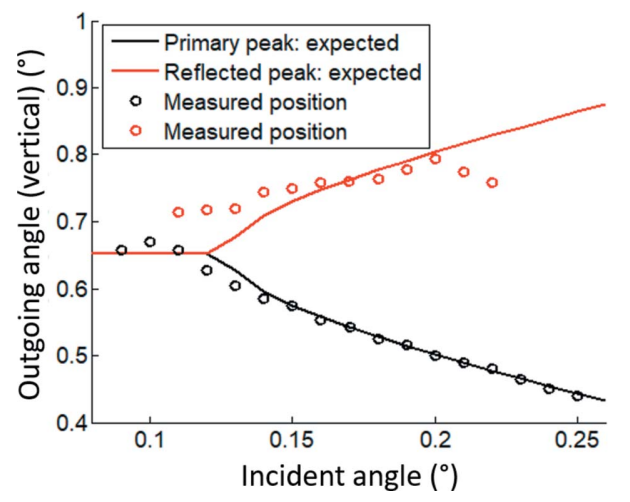

Figure 19

Comparison of our result with the experimental result obtained by Resel et al. (2016). Solid lines give our result and markers show the extracted peak positions [digitized from Resel et al. (2016)].

Resel et al. (2016) indicated that $\delta=2.48 \times 10^{-6}$ at $10.5 \mathrm{keV}$, which corresponds to a density of $1.25 \mathrm{~g} \mathrm{~cm}^{-3}$ and $\beta=$ $1.96 \times 10^{-9}$ according to our refractive index calculator (using the atomic composition $\mathrm{C}_{30} \mathrm{H}_{20}$ for $2,2^{\prime}: 6^{\prime}, 2^{\prime \prime}$-ternaphthalene). They concluded that the corrected value of $Q_{z}$ would be $0.0594 \AA^{-1}$, which we converted using the same assumption that $Q_{z}$ is directly related to $\theta_{0 \mathrm{~s}, Z}$ :

$$
\sin \frac{\theta_{1}+\theta_{0 \mathrm{~s}, Z}}{2} \simeq \frac{\lambda Q_{z}}{4 \pi} .
$$

We applied refraction and reflection as before. We show our result overlaid with the measurements from Resel et al. (2016) in Fig. 19. We observe good consistency for incident angles between 0.14 and $0.20^{\circ}$, though there is some discrepancy outside this range. Resel et al. (2016) also observed this deviation, which was assigned to the difficulty in separating the two peaks at low angles and vanishing peak intensities at high angles. We additionally hypothesize that the observed peak splitting at low angles, and especially below the critical angle where no beam penetration is expected, is due to some combination of beam divergence and nonuniformity of sample surface.

\section{APPENDIX $C$ \\ Simulation of scattering pattern from SM1}

The results of scattering simulation using SimDiffraction (Breiby et al., 2008) are shown in Fig. 20 for four different molecular configurations. Figs. 20(a)-20(c) show a planar backbone with various thiophene configurations. We performed an energy minimization on each of these three configurations using the IQMol software (http://iqmol.org/). Fig. 20(d) shows a twisted backbone configuration which was obtained by geometry optimization of the isolated molecule using a density functional theory with the wB97X-V functional (Mardirossian \& Head-Gordon, 2014) and 6-31G* basis set in the $Q$-chem ab initio quantum chemistry package (Shao et al., 2015). The C-curve configuration [Fig. 20(c)] gave the closest results to our experimental data.

\section{Acknowledgements}

We would like to thank Professor Lyudmila Slipchenko for help in generating the molecular configurations in Fig. 20. We would like to thank Alex Liebman-Peláez and Obaid Alqahtani for help in testing and debugging the software.

\section{Funding information}

This work was supported by the Office of Naval Research NDSEG fellowship (VS). Use of the Stanford Synchrotron Radiation Lightsource, SLAC National Accelerator Labora-

Figure 20

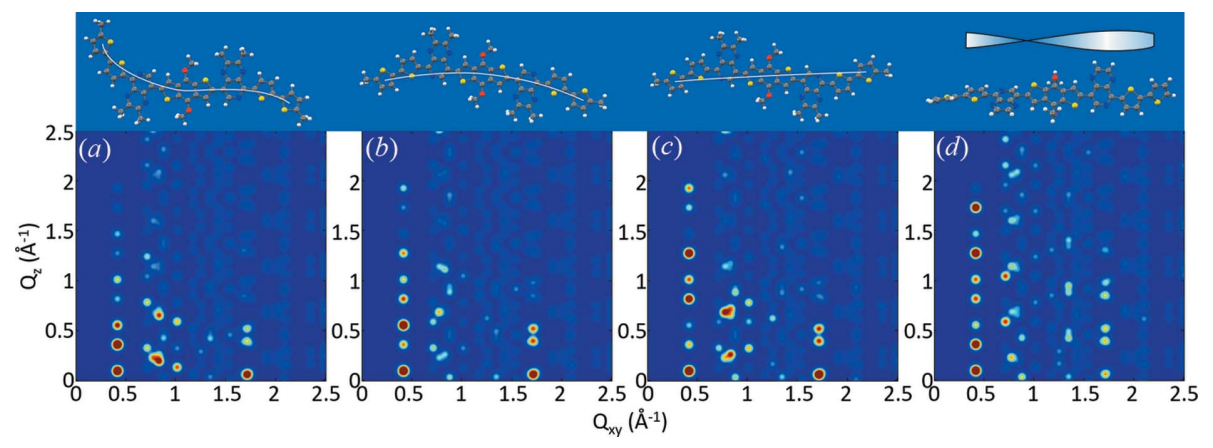

Simulated scattering patterns with four different molecular configurations, which we call $(a)$ S-curve, $(b)$ C-curve, $(c)$ straight, $(d)$ twisted. The result for the C-curve structure is also shown in Fig. 12(d). 
tory, is supported by the US Department of Energy, Office of Science, Office of Basic Energy Sciences under contract No. DE-AC02-76SF00515. The authors also acknowledge support from the Department of the Navy, Office of Naval Research award No. N00014-14-1-0580. This research was supported in part through computational resources provided by Information Technology at Purdue, West Lafayette, Indiana.

\section{References}

Akimichi, H., Inoshita, T., Hotta, S., Noge, H. \& Sakaki, H. (1993). Appl. Phys. Lett. 63, 3158-3160.

Alqahtani, O., Babics, M., Gorenflot, J., Savikhin, V., Ferron, T., Balawi, A. H., Paulke, A., Kan, Z., Pope, M., Clulow, A. J., Wolf, J., Burn, P. L., Gentle, I. R., Neher, D., Toney, M. F., Laquai, F., Beaujuge, P. M. \& Collins, B. A. (2018). Adv. Energy Mater. 8, 1702941.

Als-Nielsen, J., Jacquemain, D., Kjaer, K., Leveiller, F., Lahav, M. \& Leiserowitz, L. (1994). Phys. Rep. 246, 251-313.

Als-Nielsen, J. \& McMorrow, D. (2011). Elements of Modern X-ray Physics, 2nd ed. Chichester: John Wiley \& Sons.

Attwood, D. T. (1999). Soft X-rays and Extreme Ultraviolet Radiation: Principles and Applications. Cambridge University Press.

Bevington, P. R. \& Robinson, D. K. (2003). Editors. Data Reduction and Error Analysis for the Physical Sciences, 3rd ed. New York: McGraw-Hill.

Boer, D. K. G. de (1991). Phys. Rev. B, 44, 498-511.

Borne, M. \& Wolfe, E. (1985). Principles of Optics. Oxford: Pergamon Press.

Breiby, D. W., Bunk, O., Andreasen, J. W., Lemke, H. T. \& Nielsen, M. M. (2008). J. Appl. Cryst. 41, 262-271.

Busch, P., Rauscher, M., Moulin, J.-F. \& Müller-Buschbaum, P. (2011). J. Appl. Cryst. 44, 370-379.

Cadek, M., Vostrowsky, O. \& Hirsch, A. (2010). Carbon, 7. Fullerenes and Carbon Nanomaterials, in Ullmann's Encyclopedia of Industrial Chemistry. New York: John Wiley \& Sons.

Chabinyc, M. (2010). Gixs-calculator Tool, https://www-ssrl.slac. stanford.edu/materialscatter/gixs-calculator.xls

Chang, S.-W., Waters, H., Kettle, J. \& Horie, M. (2012). Org. Electron. 13, 2967-2974.

Chen, A. Z., Shiu, M., Ma, J. H., Alpert, M. R., Zhang, D., Foley, B. J., Smilgies, D.-M., Lee, S.-H. \& Choi, J. J. (2018). Nat. Commun. 9, 1336.

Chen, X., Rogers, J. A., Lacour, S. P., Hu, W. \& Kim, D.-H. (2019). Chem. Soc. Rev. 48, 1431-1433.

Congreve, D. N., Lee, J., Thompson, N. J., Hontz, E., Yost, S. R., Reusswig, P. D., Bahlke, M. E., Reineke, S., Van Voorhis, T. \& Baldo, M. A. (2013). Science, 340, 334-337.

Corricelli, M., Altamura, D., Curri, M. L., Sibillano, T., Siliqi, D., Mazzone, A., Depalo, N., Fanizza, E., Zanchet, D., Giannini, C. \& Striccoli, M. (2014). CrystEngComm, 16, 9482-9492.

Daillant, J. \& Gibaud, A. (2009). X-ray and Neutron Reflectivity: Principles and Applications. Berlin: Springer.

Dane, T. G., Kieffer, J. \& Lilliu, S. (2020). Github, https://github.com/ tgdane/pygix.

Dev, B. N., Das, A. K., Dev, S., Schubert, D. W., Stamm, M. \& Materlik, G. (2000). Phys. Rev. B, 61, 8462.

Factor, B. J. (1991). Grazing Incidence X-ray Scattering Studies of Thin Polymer Films. Dissertation, Stanford University, CA, USA.

Factor, B. J., Russell, T. P. \& Toney, M. F. (1993). Macromolecules, 26, 2847-2859.

Fiorito, R. B., Shkvarunets, A. G., Watanabe, T., Yakimenko, V. \& Snyder, D. (2006). Phys. Rev. A, 9, 052802.

Fritz, S. E., Martin, S. M., Frisbie, C. D., Ward, M. D. \& Toney, M. F. (2004). J. Am. Chem. Soc. 126, 4084-4085.
Gann, E., Caironi, M., Noh, Y.-Y., Kim, Y.-H. \& McNeill, C. R. (2018). Macromolecules, 51, 2979-2987.

Giri, G., Verploegen, E., Mannsfeld, S. C. B., Atahan-Evrenk, S., Kim, D. H., Lee, S. Y., Becerril, H. A., Aspuru-Guzik, A., Toney, M. F. \& Bao, Z. (2011). Nature, 480, 504-508.

Hailey, A. K., Hiszpanski, A. M., Smilgies, D.-M. \& Loo, Y.-L. (2014). J. Appl. Cryst. 47, 2090-2099.

Hartzler, D. A., Slipchenko, L. V. \& Savikhin, S. (2018). J. Phys. Chem. A, 122, 6713-6723.

Henke, B. L., Gullikson, E. M. \& Davis, J. C. (1993). At. Data Nucl. Data Tables, 54, 181-342.

Hernandez, J. L., Deb, N., Wolfe, R. M. W., Lo, C. K., Engmann, S., Richter, L. J. \& Reynolds, J. R. (2017). J. Mater. Chem. A, 5, 20687.

Hexemer, A. \& Müller-Buschbaum, P. (2015). IUCrJ, 2, 106-125.

Janssen, H. (2013). Reliab. Eng. Syst. Saf. 109, 123-132.

Jiang, Z. (2015). J. Appl. Cryst. 48, 917-926.

Jiang, Z., Lee, D. R., Narayanan, S. \& Wang, J. (2011). Phys. Rev. B, 84, 075440.

Johnson, B. (2012). SSRL Scattering Beam Lines, 6th SSRL School on Synchrotron, Menlo Park, CA, USA, pp. 1-23.

Kim, C., Liu, J., Lin, J., Tamayo, A. B., Walker, B., Wu, G. \& Nguyen, T.-Q. (2012). Chem. Mater. 24, 1699-1709.

Kim, H. J., Lee, H. H., Kim, J. W., Jang, J. \& Kim, J.-J. (2012). J. Mater. Chem. 22, 8881-8886.

Kippelen, B. \& Brédas, J.-L. (2009). Energy Environ. Sci. 2, 251261.

Klockenkàmper, R., Knoth, J., Prange, A. \& Schwenke, H. (1992). Anal. Chem. 64, 1115A-1123A.

Koehler, E., Brown, E. \& Haneuse, S. J.-P. A. (2009). Am. Stat. 63, 155-162.

Köhler, A. \& Bässler, H. (2009). Mater. Sci. Eng. Rep. 66, 71-109.

Liang, R.-Z., Babics, M., Savikhin, V., Zhang, W., Corre, V. M. L., Lopatin, S., Firdaus, Y., Liu, S., McCulloch, I., Toney, M. F. \& Beaujuge, P. M. (2018). Adv. Energy Mater. 8, 1800264.

Lide, D. R. (2005). Editor. Physical Constants of Inorganic Compounds, in CRC Handbook of Chemistry and Physics, Internet Version 2005. Boca Raton: CRC Press.

Lilliu, S., Griffin, J., Barrows, A. T., Alsari, M., Curzadd, B., Dane, T. G., Bikondoa, O., Macdonald, J. E. \& Lidzey, D. G. (2016). CrystEngComm, 18, 5448-5455.

Liman, C. D., Choi, S., Breiby, D. W., Cochran, J. E., Toney, M. F., Kramer, E. J. \& Chabinyc, M. L. (2013). J. Phys. Chem. B, 117, 14557-14567.

Lin, Y.-H., Yager, K. G., Stewart, B. \& Verduzco, R. (2014). Soft Matter, 10, 3817-3825.

Liu, F., Gu, Y., Shen, X., Ferdous, S., Wang, H.-W. \& Russell, T. P. (2013). Prog. Polym. Sci. 38, 1990-2052.

Liu, J., Haynes, D., Balliet, C., Zhang, R., Kowalewski, T. \& McCullough, R. D. (2012). Adv. Funct. Mater. 22, 1024-1032.

Liu, J. \& Yager, K. G. (2018). IUCrJ, 5, 737-752.

Mannsfeld, S. C. B., Tang, M. L. \& Bao, Z. (2011). Adv. Mater. 23, 127-131.

Mardirossian, N. \& Head-Gordon, M. (2014). Phys. Chem. Chem. Phys. 16, 9904-9924.

Mateker, W. R., Heumueller, T., Cheacharoen, R., Sachs-Quintana, I. T. \& McGehee, M. D. (2015). Chem. Mater. 27, 6345-6353.

Medvedovski, E., Alvarez, N., Yankov, O. \& Olsson, M. K. (2008). Ceram. Int. 34, 1173-1182.

Merlo, J. A., Newman, C. R., Gerlach, C. P., Kelley, T. W., Muyres, D. V., Fritz, S. E., Toney, M. F. \& Frisbie, C. D. (2005). J. Am. Chem. Soc. 127, 3997-4009.

Miller, N. C., Sweetnam, S., Hoke, E. T., Gysel, R., Miller, C. E., Bartelt, J. A., Xie, X., Toney, M. F. \& McGehee, M. D. (2012). Nano Lett. 12, 1566-1570.

Mukherjee, S., Herzing, A. A., Zhao, D., Wu, Q., Yu, L., Ade, H., DeLongchamp, D. M. \& Richter, L. J. (2017). J. Mater. Res. 32, 1921-1934.

Müller-Buschbaum, P. (2014). Adv. Mater. 26, 7692-7709. 
Nabok, D., Puschnig, P., Ambrosch-Draxl, C., Werzer, O., Resel, R. \& Smilgies, D.-M. (2007). Phys. Rev. B, 76, 235322.

Noriega, R., Rivnay, J., Vandewal, K., Koch, F. P. V., Stingelin, N., Smith, P., Toney, M. F. \& Salleo, A. (2013). Nat. Mater. 12, 10381044.

Olivier, Y., Lemaur, V., Brédas, J. L. \& Cornil, J. (2006). J. Phys. Chem. A, 110, 6356-6364.

Oosterhout, S. D., Savikhin, V., Zhang, J., Zhang, Y., Burgers, M. A., Marder, S. R., Bazan, G. C. \& Toney, M. F. (2017). Chem. Mater. 29, 3062-3069.

Osaka, I. \& Takimiya, K. (2015). Polymer, 59, A1-A15.

Parratt, L. G. (1954). Phys. Rev. 95, 359-369.

Rabedeau, T. (2006). Sources and Beam Line Optics, SSRL Scattering Workshop, Menlo Park, CA, USA, pp. 1-12.

Ran, N. A., Roland, S., Love, J. A., Savikhin, V., Takacs, C. J., Fu, Y.-T., Li, H., Coropceanu, V., Liu, X., Brédas, J.-L., Bazan, G. C., Toney, M. F., Neher, D. \& Nguyen, T.-Q. (2017). Nat. Commun. 8, 79.

Renaud, G., Lazzari, R. \& Leroy, F. (2009). Surf. Sci. Rep. 64, 255-380.

Resel, R., Bainschab, M., Pichler, A., Dingemans, T., Simbrunner, C., Stangl, J. \& Salzmann, I. (2016). J. Synchrotron Rad. 23, 729-734.

Rivnay, J., Noriega, R., Kline, R. J., Salleo, A. \& Toney, M. F. (2011). Phys. Rev. B, 84, 045203.

Sasaki, S., Masunaga, H., Tajiri, H., Inoue, K., Okuda, H., Noma, H., Honda, K., Takahara, A. \& Takata, M. (2007). J. Appl. Cryst. 40, s642-s644.

Savikhin, V., Babics, M., Neophytou, M., Liu, S., Oosterhout, S. D., Yan, H., Gu, X., Beaujuge, P. M. \& Toney, M. F. (2018). Chem. Mater. 30, 7872-7884.

Savikhin, V., Jagadamma, L. K., Purvis, L. J., Robertson, I., Oosterhout, S. D., Douglas, C. J., Samuel, I. D. W. \& Toney, M. F. (2018). iScience, 2, 182-192.

Savikhin, V. \& Toney, M. F. (2019). Handbook of Organic Materials for Electronic and Photonic Devices, 2nd ed., ch. 15, edited by O. Ostroverkhova. Duxford, Cambridge, Kidlington: Woodhead Publishing.
Servet, B., Ries, S., Trotel, M., Alnot, P., Horowitz, G. \& Garnier, F. (1993). Adv. Mater. 5, 461-464.

Shao, Y. et al. (2015). Mol. Phys. 113, 184-215.

Sherman, J. B., Purushothaman, B., Parkin, S. R., Kim, C., Collins, S., Anthony, J., Nguyen, T.-Q. \& Chabinyc, M. L. (2015). J. Mater. Chem. A, 3, 9989.

Siliqi, D., De Caro, L., Ladisa, M., Scattarella, F., Mazzone, A., Altamura, D., Sibillano, T. \& Giannini, C. (2016). J. Appl. Cryst. 49, $1107-1114$

Simbrunner, J., Simbrunner, C., Schrode, B., Röthel, C., BedoyaMartinez, N., Salzmann, I. \& Resel, R. (2018). Acta Cryst. A74, 373-387.

Skinner, L. B., Benmore, C. J. \& Parise, J. B. (2012). Nucl. Instrum. Methods Phys. Res. Sect. A, 662, 61-70.

Smilgies, D.-M. (2009). J. Appl. Cryst. 42, 1030-1034.

Steinrück, H.-G., Magerl, A., Deutsch, M. \& Ocko, B. (2014). Phys. Rev. Lett. 113, 156101.

Stepanov, S. (2019). TER_sl on the Web, https://x-server.gmca.aps. anl.gov/TER_sl.html.

Stepanov, S. A. (2004). Proc. SPIE, 5536, 16-26.

Stepanov, S. A., Kondrashkina, E. A., Köhler, R., Novikov, D. V., Materlik, G. \& Durbin, S. M. (1998). Phys. Rev. B, 57, 48294841.

Stribeck, N. \& Nöchel, U. (2009). J. Appl. Cryst. 42, 295-301.

Toby, B. H. \& Von Dreele, R. B. (2013). J. Appl. Cryst. 46, 544-549.

Toney, M. F. \& Brennan, S. (1989). Phys. Rev. B, 39, 7963-7966.

Vineyard, G. H. (1982). Phys. Rev. B, 26, 4146-4159.

Watts, B. (2014). Opt. Express, 22, 23628-23639.

Waldemar Knittel Glasbearbeitungs (2019). Microscope Slides - Data Sheet, http://www.knittelglass.com.

Wolf, J., Babics, M., Wang, K., Saleem, Q., Liang, R.-Z., Hansen, M. R. \& Beaujuge, P. M. (2016). Chem. Mater. 28, 2058-2066.

Yang, J., De, S., Campbell, J. E., Li, S.,Ceriotti, M. \& Day, G. M. (2018). Chem. Mater. 30, 4361-4371.

Zhong, Y., Munir, R., Li, J., Tang, M.-C., Niazi, M. R., Smilgies, D.-M., Zhao, K. \& Amassian, A. (2018). ACS Energy Lett. 3, 1078-1085. 